\title{
Online measures of looking and learning in infancy
}

Alexis S. Smith-Flores ${ }^{1,2}$, Jasmin Perez ${ }^{2}$, Michelle H. Zhang ${ }^{2}$, and Lisa Feigenson ${ }^{2}$

${ }^{1}$ Department of Psychology, University of California - San Diego 9500 Gilman Dr., La Jolla, CA 92093

${ }^{2}$ Department of Psychological and Brain Sciences, Johns Hopkins University 3400 N. Charles Street, Baltimore, MD 21218

Word Count: 8,927

\section{ORCID ID}

Alexis S. Smith-Flores: https://orcid.org/0000-0002-6755-4986

Jasmin Perez: https://orcid.org/0000-0003-1543-5755

Michelle H. Zhang: https://orcid.org/0000-0001-9186-6712

Lisa Feigenson: https://orcid.org/0000-0002-9015-7065

\section{Data Availability Statement}

The data that support the findings of this study are available on request from the corresponding author.

\section{Funding Statement}

This research received no specific grant from any funding agency in the public, commercial, or not-for-profit sectors.

\section{Ethics Approval Statement}

This study was conducted with approval from the Johns Hopkins University Institutional Review Board (Approval \#HIRB00009205).

\section{Conflict of Interest}

The authors declare no conflicts of interest. 


\begin{abstract}
Infants in laboratory settings look longer at impossible than possible events, learn better about objects that behave surprisingly, and match people's utterances to the objects that likely elicited them. The paradigms that reveal these behaviors have become cornerstones of research on preverbal cognition. But less is known about whether these canonical behaviors generalize in naturalistic environments. Here we describe a series of online protocols that replicate classic laboratory findings, detailing our methods throughout. In Experiment 1, we found that 15-month-olds $(N=24)$ looked longer at an online impossible support event (an object appearing to defy gravity) than a possible support event. These infants did not, however, show the same success with an online solidity event. In Experiment 2, we found that 15-montholds $(N=24)$ showed surprise-induced learning online-they were better able to learn a label for a novel object when the object had just behaved unexpectedly. Finally, in Experiment 3, we found that 16 -month-olds $(N=20)$ who heard a valenced utterance ("Yum!") showed preferential looking to the object most likely to have generated that utterance. Together, these results suggest that, with some adjustments, testing infants online is a feasible and promising approach for cognitive development research.
\end{abstract}

Keywords: infants, violation-of-expectation, looking time, online testing, research methods 


\section{Online measures of looking and learning in infancy}

For several decades, infants' looking responses in laboratory experiments have provided a window on early human cognition and perception (see, e.g., Aslin, 2007; Spelke \& Kinzler, 2007). Measuring what infants look at, when, and for how long, has enabled researchers to make inferences about early-emerging abilities and expectations, long before infants can engage in tasks that require language or instruction. Experiments measuring looking time employ a range of paradigms, including violation-of-expectation, habituation, anticipatory looking, object-sound matching, and preferential looking, and have yielded rich information regarding what infants know about objects, quantities, language, categories, and other people.

An outstanding question for developmental psychology concerns the generality of these responses: can infant looking times only be reliably captured in the laboratory, or can they also be obtained using virtual methods, in naturalistic settings? This question is especially pressing at present, as the COVID-19 global pandemic has shuttered research laboratories around the world. In-person infant testing has largely ceased, for now. Must infant looking-time research also be put on hold?

Even before the first news of COVID-19, efforts were being made to develop purely online platforms for conducting research with infants and young children. In one especially broadly-scaled effort, Scott and Schulz (2017) created an automated online platform called Looklt, designed to allow researchers to test participants asynchronously. To participate in a Looklt experiment, parents log on to the website at their convenience, without the presence or assistance of an experimenter, and follow the posted instructions to initiate the test stimuli. The webcam in parents' computers transmit video of infants' looking or children's responses, which can be coded later by the research team. This automation feature necessitates that experiments on Looklt be self-explanatory in their set-up, and robust across a range of parameters (e.g., participants' screen size, viewing distance and angle, lighting conditions, audio quality). Scott and colleagues reported an initial comparison of data collected using Looklt, relative to in-lab 
experiments, in three experiments involving violation-of-expectation (VOE) with 14-month old infants, preferential looking with 2-year olds, and production of a novel label with 4-year olds (Scott, Chu, \& Schulz, 2017). The first two are most relevant to the present work, as they measured looking time. In the violation-of-expectation experiment, adapted from Téglás and colleagues (2007), infants watched four objects bouncing in a container-- three were identical and one differed in shape and color. The container was then covered, and then one of the objects "randomly" emerged from an opening in the bottom. In the original study, 12-month-old infants looked longer when the unique object emerged, compared to one of the majority objects. Scott et al.'s (2017) replication also found longer looking to this improbable outcome compared to the probable one; however, the difference was considerably smaller than the in-lab study and did not reach statistical significance (even with more than double the sample size of the original study). In the preferential looking experiment, based on Yuan and Fisher (2009), 2-year-olds' syntactic bootstrapping abilities were tested by presenting them with a video of an action involving a single person and a video of an action involving two people, and asking them to find a novel verb (e.g., "Find blicking!"), that earlier had been used in either a transitive or an intransitive sentence. Children in Scott and colleagues' online replication showed similar looking patterns to children in Yuan and Fisher's in-person study. Overall, these results suggest that positive looking-time results can be obtained with toddlers, but leave open whether looking-time studies with infants can be run successfully online.

Outside of the Looklt platform, other researchers have used Amazon Mechanical Turk (MTurk) for online infant recruitment and testing. Tran, Cabral, Patel, and Cusack (2017) showed infants various clips from children's television programs and measured their looking using automated online testing without an experimenter, similar to Looklt. Rather than seeking to replicate any particular in-lab finding, Tran and colleagues sought to determine simply whether parents would volunteer their infants to participate in a study on MTurk, whether infants would be engaged by videos on a home computer, and whether the online recordings of infants' 
looking would be of sufficient quality to support later coding. They found were able to capture infants' looking time, and that most infants in their sample were engaged by the stimuli (and by some of the videos in particular). Tran et al. also noted that although data collection proceeded more quickly than in-person data collection (147 5- to 8-month-olds tested over a two-week period), $61.2 \%$ of their sample (90 infants) had to be excluded because of technical issues (e.g., low video quality, poor infant placement in the video). The high exclusion rate was similar to that in Scott et al.'s Looklt study (2017), in which data from $58.4 \%$ of infants in the VOE experiment (157 of 269 infants) and $57.4 \%$ of toddlers in the preferential looking experiment (189 of 329 toddlers) were excluded from the final analysis.

No other published studies that we are aware of have tested infants' looking time online. However, there are other online studies with older children, using measures other than looking. Rhodes and colleagues (Rhodes et al., 2020) developed an unmoderated platform similar to Looklt, in that parents and children participate without guidance from a live experimenter. They report a replication of a study by Rhodes, Leslie, and Tworek (2012), which asked whether young school-age children (4- to 8-years) exhibit an increase in essentialist beliefs when hearing statements using generic language. In their large, multi-national sample, they observed a similar pattern of results to the original, in-person study.

Successful online testing of children was also reported by Sheskin and Keil (2018), whose online video chat platform, The Child Lab, is run using Adobe Flash with a live experimenter. Sheskin and Keil tested their platform using a false belief task (Baron-Cohen et al., 1985), a resource distribution task assessing children's intuitions about fairness (Sheskin et al., 2016), and a plinko machine task in which children tried to predict object trajectories (Hood, 1995). Overall, the 50 children tested (ranging from 4- to 12-years old) performed well in the fairness and plinko tasks (at least 46 children answering correctly in each), but were less successful in the false belief task, with only 33 of them correctly identifying where a character with a false belief would search for a hidden object. Although this pattern of results suggests 
that online testing, though feasible, was not equivalent to in-person testing, it is worth nothing that children succeeded in two of the three tasks, and that no children had to be excluded from Sheskin and Keil's analysis, which suggests either that testing with a live experimenter or testing of older children may result in lower exclusion rates.

The recent advances in online testing reviewed above suggest that it may be possible to capture infants' looking patterns online, in their own homes, even in the midst of a pandemic. However, an outright success at using online methods to replicate a classic infant looking-time result has yet to be observed. The "live experimenter" methods of Sheskin and Keil (2018) lend credence to the idea that laboratory tasks can be at least partially recreated off-campus, retaining the social interaction with the experimenter that may help children understand the task or feel engaged, and/ or help parents adjust their cameras or computers to optimize data transmission. Our goal in the present work was to develop a protocol that could feasibly merge these two ideas: testing infants online, using a live video-chat method.

In three experiments, we tested infants in three classic infant looking-time paradigms, online with a live experimenter. Experiment 1 used an adapted version of the violation-ofexpectation (VOE) paradigm based on previous work by Needham and Ballargeon (1993) and Spelke, Breinlinger, Macomber, and Jacobson (1992). We showed infants two events: one involving object support and the other involving object solidity. Each infant saw one event culminate in an expected outcome (i.e., an object moved while remaining fully supported, or a moving object was stopped by a wall in its path), and one event culminate in a surprising outcome (i.e., an object moved completely off its supporting surface and appeared to hover in mid-air, or an object appeared to pass straight through a wall in its path). Based on previous work, we expected infants to look longer at the surprising than the expected outcomes. In Experiment 2, we measured infants' learning following object events with expected versus surprising outcomes, as in laboratory experiments by Stahl and Feigenson (2015). Following the expected or surprising object event and the measurement of their looking at the event outcome, 
infants from Experiment 1 were taught a novel label for the object (that had just accorded with or defied expectations). Then their learning of the novel label was measured. Based on previous work, we expected infants to learn better following surprising events. Finally, in Experiment 3, we tested infants in a preferential looking time paradigm similar to that of $\mathrm{Wu}$, Muentener, and Schulz (2017), which measured infants' looking following valenced utterances. Infants heard an experimenter say either "Yum!" or "Oh!" (a neutral utterance) while they saw an appetizing stimulus (like cake) and a positive neutral stimulus (a toy). Based on previous work, we expected infants to increase their looking to the appetizing stimulus when hearing "Yum!" but not "Oh!".

\section{General Method}

\section{Recruitment}

Participants were recruited from a database of children whose parents had previously expressed interest in laboratory research opportunities via local recruitment (e.g., farmers' markets, preschools, museums) and online advertising. Parents of infants between 13- and 19months of age received an email inviting them to participate in a 20-minute online research study with their child. After parents had scheduled a virtual appointment, they received an email with a meeting link, a separate link to an online consent form, and a link to an optional demographics form. Parents were told that they could wait to sign the consent form until their appointment if they had any questions. All forms were hosted and completed through Qualtrics. Parents were emailed a $\$ 5$ Amazon gift card after their visit.

\section{Consent}

Parents signed an electronic consent form approved by the university Internal Review Board (IRB); at the end of the form parents typed their full legal name to give consent for their child to participate and for the testing session to be recorded. The form also included a PDF version of the consent form that parents could download if they wished. The last question on the 
form asked about the type of computer parents would use during the visit in order to collect data about screen size constraints.

When the online appointment began, the experimenter introduced themselves and briefly explained the study's aim and methods. If parents had not already signed the consent form, the experimenter asked if the parent had questions about the form or the study, and then, when parents were ready, resent the consent form link. If parents had already signed the consent form, the experimenter asked if they had any further questions, reiterated that the study would be video recorded, and reminded parents that the study could be stopped at any time.

Online study recordings were saved directly to the lab's secure video server, which was only accessible through the university's virtual private network (VPN).

\section{Online Protocol}

\section{Video Chat Set-Up}

Given the importance of capturing fine-grained eye movements, and previous reports of high numbers of participant exclusions in other online studies (e.g., Scott et al. 2017; Tran et al., 2017), our primary concern about online testing was capturing high quality data. To maximize data quality, we designed an online testing protocol that allowed the experimenter to troubleshoot in real time, just as they would if the study were run in a campus laboratory. Using a live experimenter gave parents the opportunity to ask questions and allowed the experimenter to help parents modify their set up. For example, the experimenter sometimes asked parents to move infants closer to or farther from the screen, adjust the lighting, or move infants from a highchair to the parent's lap if they become fussy. These modifications helped the experimenter capture higher quality data. Additionally, the live format of the experiment allowed the experimenter to pause the testing session if infants became upset. Depending on when the pause occurred (e.g., during a pre-trial attention getter or between test blocks), the study could resume after infants had calmed down or parents readjusted. Because of the variety of screen 
sizes across households, a calibration video was included at the beginning of each testing session to capture footage of infants' looks to the center, left, and right sides of their screen.

\section{Zoom Meeting Setup}

We chose Zoom as our video chat platform as many parents were anticipated to already be familiar with it. We pre-tested the quality of our stimulus videos via Zoom screen-sharing amongst research team members, in order to determine whether they were of sufficient quality for online testing. ${ }^{1}$ Prior to each Zoom testing session, we set up the recording output so that it would capture both the video stimuli and an image of the participant. Additionally, our settings enabled the footage of participants to be positioned to the right of the stimulus footage, and for the entire recording to be saved directly to the lab's secure video server rather than on a personal computer. For details on the settings we used, see the Supplementary Materials.

Once on the Zoom call, parents were provided more details about the study. The experimenter then prompted parents to position infants in a highchair, or on their lap if a highchair was not available or suitable for the position of the parent's computer. Parents were further instructed to remain silent throughout the study and to avoid interacting with infants (e.g., not to comment, point at the screen, or otherwise redirect infants' attention). Once infants were positioned close enough to the screen to capture their gaze, but far away enough so that infants could not reach the keyboard or computer mouse, the experimenter began recording and initiated the stimulus video.

\section{Exclusion Criteria}

\footnotetext{
${ }^{1}$ We highly recommend pre-testing stimuli online prior to the actual study. If, after testing video stimuli on Zoom via screen-sharing, the videos are judged to be of poor quality (e.g., the video lags, images are distorted, sound is not synced) an alternative platform for hosting stimuli is slides.com (Slides). Slides supports online slide show presentation, with video stimuli uploaded to the website, inserted onto a series of slides, and displayed live. The benefit of this format is that the presented videos are of higher quality, with reduced lags between video and audio. In addition, although we do not discuss infant controlled paradigms here, Slides can be enabled for presenting stimuli using an infant controlled procedure rather than fixed looking windows. Zoom can be modified to record studies that use Slides to present stimuli (see Supplemental Materials for manual on Zoom setup).
} 
Participants were excluded for the same reasons as would apply during laboratory testing (e.g., fussiness, parental interference, experimenter error). We also excluded infants who looked less than two seconds during any measurement period (indicating a general lack of interest in the stimuli) or did not look within the first five seconds of the event looking window (see Experiments 1-3, below). Two additional exclusion criteria were added as a result of online testing; these concerned video quality and screen size. First, infants' data were excluded if their video recording had a noticeable lag between the stimuli's audio and video (as it became difficult to know when coding for a particular measurement period should begin), or if their video recordings were of a quality that was too poor to clearly see where infants were looking. Additionally, we excluded any participant whose screen size measured less than 10 inches or greater than 24 inches diagonally. Screens that were too small precluded us from being able to accurately code where infants were looking; screens that were too large required infants to turn their heads to see some of the presented stimuli, thereby also making coding difficult.

\section{Experiment 1: Violation-of-Expectation in Solidity and Support Events}

Experiment 1 sought to determine whether infants would show the classic pattern of heightened looking in response to a violation-of-expectation (VOE), in this case involving the behavior of physical objects. We tested 15-month-old infants, using an adapted version of the VOE paradigm in the first experiment by Stahl and Feigenson (2015). Infants' looking was compared for events in which expectations about object support were or were not violated, and events in which expectations about object solidity were or were not violated.

\section{Method}

\section{Participants}

Twenty-four 13- to 19-month-old infants participated (Mage: 15.68 months, range: 13.15 months to 19.00 months, SD: 1.75 months; 13 girls). We tested infants across a relatively wide range of ages in order to begin to assess the generality of our online testing results. Nineteen infants sat in a highchair and five sat on their caregiver's lap. Data from 25 additional infants 
were excluded for screen size constraints (4), fussiness (7), interference from a household member (5), low quality video (3), or looking time during event windows being less than two seconds (6). One child was identified by their caregiver as Black or African American, 19 as White, and four as belonging to two or more races. Two caregivers indicated their child was Hispanic/ Latinx. The average screen size across participants was 13.7 inches (range: 11 inches to 21.5 inches).

\section{Design and Stimuli}

Each infant participated in a total of two trials, one involving a solidity event and one involving a support event. One of these events ended in an expected outcome and the other in a surprising outcome. Whether the surprising outcome was associated with a support or a solidity event was counterbalanced across infants, as was whether infants saw the support event first or second. This yielded four possible combinations of event outcome pairings and orders.

In the support event, infants saw two novel objects, both easily visually distinguishable from one another: a round green dog toy and a long orange dog toy. The green toy measured 8 $\mathrm{cm}$ in diameter and the orange toy measured $15 \mathrm{~cm}$ long $\times 7 \mathrm{~cm}$ wide. Which of these served as the Target Object and which served as the Distractor Object was counterbalanced across participants.

In the solidity event, infants saw two novel objects with hidden wheels (so that they could be rolled). One had a yellow wicker top with a pink felt belt around its lower half $(16.5 \times 13 \times$ $14.5 \mathrm{~cm}$ ), and the other was made of grey felt and had a pink hair curler positioned vertically on top with a blue pipe cleaner wrapped around its perimeter $(17 \times 10 \times 16 \mathrm{~cm})$. For half the infants, the yellow wicker object was the Target Object and the grey object was the Distractor, and for the other half this pairing was reversed.

Infants saw the digitally recorded events unfold on a black puppet stage. A black curtain was lowered to cover the stage between trials. 


\section{Procedure}

Calibration. The study began with a brief calibration event in which an image of a colorful beach ball bounced in the middle of the screen, then a fish bounced on the left side of the screen, and finally a different fish bounced on the right side of the screen (in the locations where the test stimuli would later appear). Each bounce was accompanied by a Boing! sound to draw infants' attention. This calibration window provided coders with an image of what it looked like when each infant was looking in the key locations of the screen.

Support Event. During the support event, infants first saw a black curtain raised to reveal the novel green and orange objects stationary on a puppet stage, with a black occluding screen resting between them. The experimenter pointed to both objects simultaneously, one with each hand, and said, "Look at these!" She then grasped an object in each hand, picked them up, and placed them down again on the stage. The video zoomed in on each object so infants could see it up close. Then the curtain was lowered; this marked the end of the 20second object familiarization event. Infants next saw the curtain raised again to reveal each object positioned atop a gray block on either side of the stage. Whether the Target Object was on the left or right was counterbalanced across participants. The experimenter said, "Look at this!" as she reached down and touched the Target Object with her index finger (Figure 1, Panel 1). The experimenter then either pushed the Target Object to the edge of the block, with the object remaining fully supported as it moved (Expected Outcome; Figure 1, Panel 2A) or pushed the Target Object completely off the block, leaving it suspended in mid-air (Surprising Outcome; Figure 1, Panel 2B). Infants' looking at the outcome after the experimenter removed her finger was measured for 15 seconds.

\section{Figure 1}

Schematic of Experiment 1 support event 


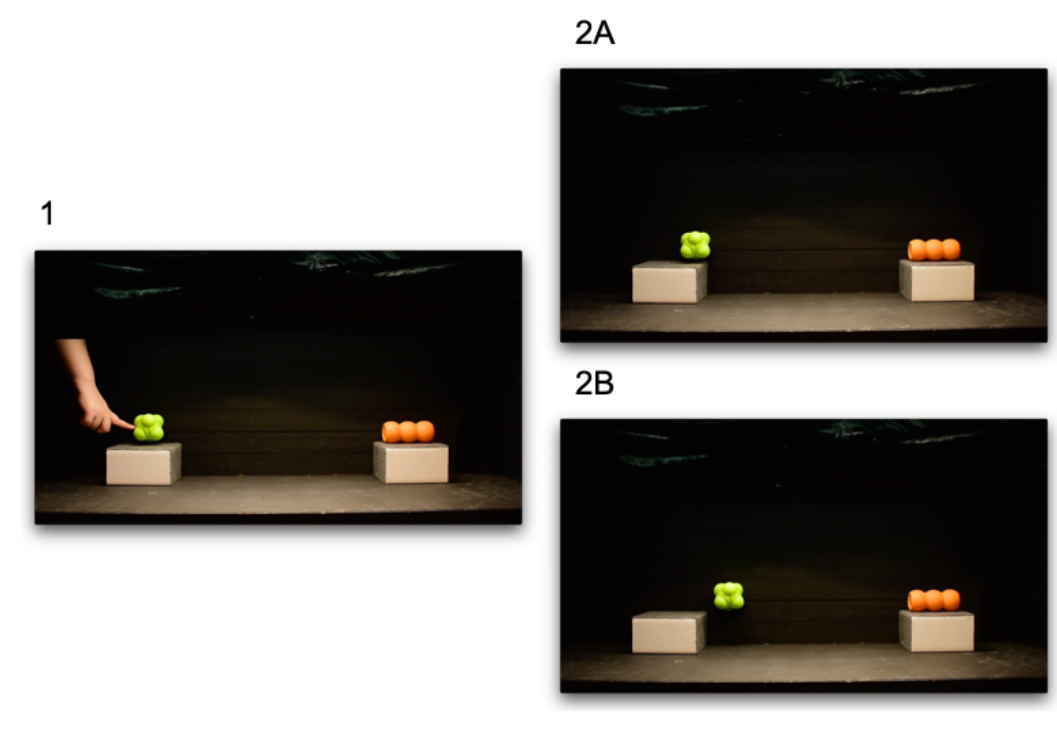

Note. The experimenter drew infants' attention to one of the novel objects (Panel 1). The experimenter then either pushed it to the edge of the block, leaving it supported (Expected Outcome, Panel 2A) or pushed it off the block completely, leaving it apparently floating in midair (Surprising Outcome, Panel 2B).

Solidity Event. The solidity Event began with a short familiarization trial, designed to introduce infants to the stimulus objects and the ramp. Infants first saw two novel objects on an empty stage. As in the support event, the camera objects zoomed in on each object, to show them to infants up close. Then the curtain was lowered; this marked the end of the 20-second object familiarization event. Next, infants saw the curtain raised to reveal a black ramp $(120 \mathrm{~cm}$ long) slanting downward from the left side of the puppet stage. At the opposite end of the stage was a grey wall. Infants saw the experimenter reach down and place a black occluding screen on stage, partially concealing the path between the end of the ramp and the grey wall. The curtain was lowered, and then immediately raised to reveal the two objects onstage: the Target Object atop the ramp, and the other object resting stationary in the left corner of the stage. The experimenter said, "Look at this!", reached down and pointed to the Target Object, then said, "Watch this!" and pushed it so that it rolled down the ramp and behind the occluding screen. She then said, "Look at this!" as she removed the screen, revealing the Target Object resting 
against the grey wall on the far right side of the stage. After five seconds, the experimenter said, "Down we go!" and lowered the curtain.

Next, in the critical event trial, the curtain was again raised to reveal the ramp, the black occluding screen covering part of the trajectory from the end of the ramp to the right end of the stage, and both objects in the same positions they had been in during familiarization (the Target Object atop the ramp and the other object on the left side of the stage floor). In addition, a highly salient red wall could be seen protruding $13 \mathrm{~cm}$ above the top of the occluding screen (Figure 2, Panel 1). The experimenter drew attention to the red wall by running her finger down its side while saying, "Look at this!" Next, she said, "Watch this!" and pushed the Target Object so that it rolled down the ramp and passed behind the occluding screen. After two seconds, the experimenter said, "Look at this!" as she removed the occluding screen to reveal either the Target Object resting against the red wall -as if the red wall had stopped the Target Object (Expected Outcome; Figure 2, Panel 2A) - or against the far grey wall -as if it had passed through the red wall (Surprising Outcome; Figure 2, Panel 2B). Infants' looking was measured for 15 seconds after the occluding screen was removed.

\section{Figure 2}

Schematic of Experiment 1 solidity event 


\section{$2 \mathrm{~A}$}

1
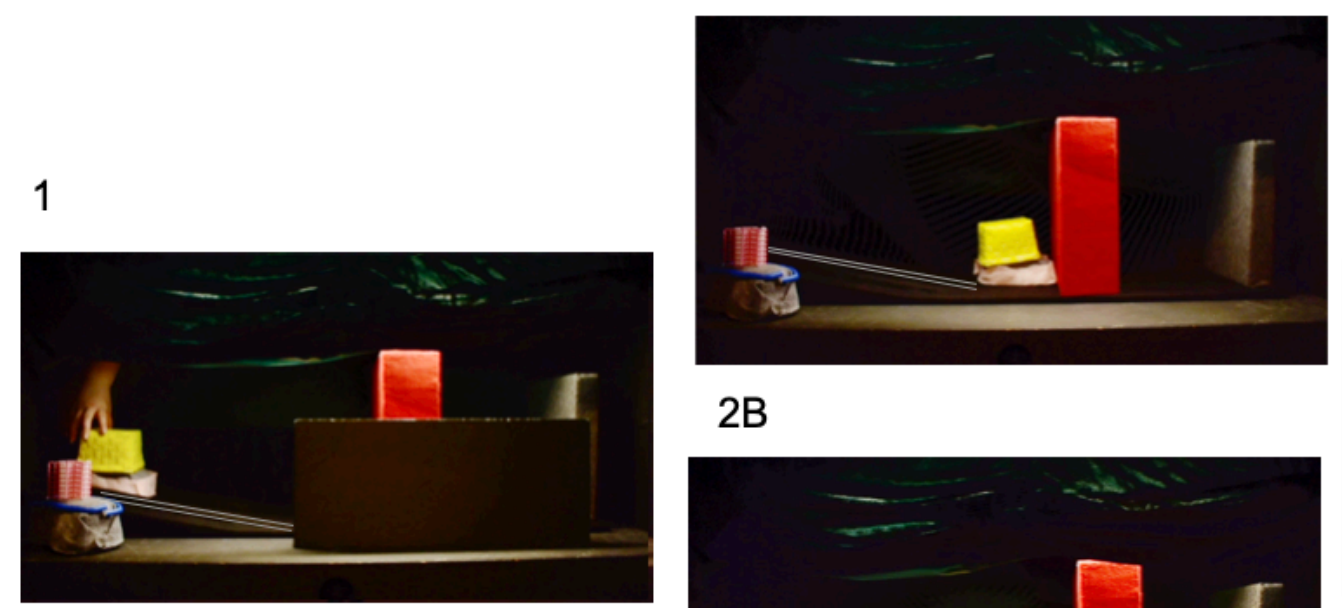

2B

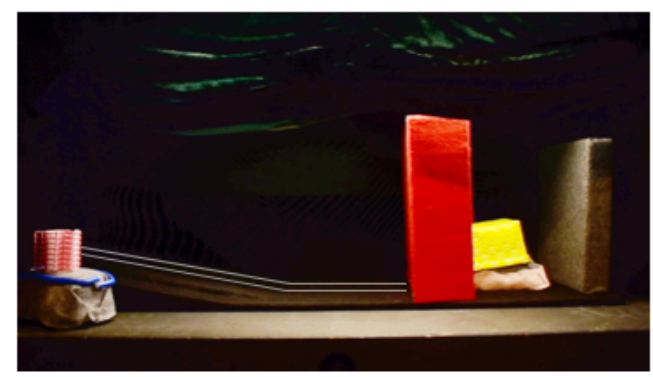

Note. Infants saw the Target Object atop the ramp and the other object sitting nearby on

(Panel 1). After the Target Object was pushed down the ramp, the black occluding screen was raised to reveal the Target Object on the near side of the red wall (Expected outcome, Panel 2A) or on the far side of the red wall (Surprising Outcome, Panel 2B).

\section{Results}

Infants' looking at the event outcomes was coded offline by two experienced observers. Twenty percent of the video footage was coded by both observers; their intercoder reliability was $95.6 \%$. We examined infants' looking using a repeated measures ANOVA with Outcome Type as a within-subject factor (Surprising vs. Expected), and Outcome Event Pairing (whether infants saw a Solidity event with a Surprising Outcome and a Support event with an Expected Outcome, or a Solidity event with an Expected Outcome and a Support event with a Surprising Outcome) and Outcome Order (Surprising Outcome or Expected Outcome first) as betweensubject factors. This revealed no main effect of Outcome Type, $F(1,20)=1.44, p=.24, \eta^{2}=$ .07 , nor of Outcome Event Pairing, $F(1,20)=2.58, p=.124, \eta^{2}=.11$, or Outcome Order, $F(1$, 20) $=0.45, p=.51, \eta^{2}=.02$. However, we observed a marginally significant interaction between 
looking at the Outcome Type and Outcome Event Pairing, $F(1,20)=3.35, p=0.08, \eta^{2}=.14$ : the difference in infants' looking at Surprising versus Expected Outcomes was marginally greater for infants who saw the Support event culminate in a Surprising Outcome, compared to infants who saw the Solidity event culminate in a Surprising Outcome (Figure 3). No other interactions were significant. We further examined infants' looking using planned t-tests. We found that infants looked longer at the Surprising than the Expected outcome of the support event, $t(46)=-2.034, p=.048$; in contrast, they did not show this elevated looking to the Surprising outcome of the solidity event, $t(46)=-0.961, p=.341$.

The results of Experiment 1 offer evidence that violation-of-expectation studies can be successfully run online. Infants looked longer when an object appeared to hover in mid-air, compared to when the object remained fully supported. This success was observed despite the fact that infants received just a single opportunity to observe the support event, rather than the multiple trials that are typical of laboratory studies (e.g., Needham \& Baillargeon, 1993). However, contrary to our predictions, we did not observe a successful VOE response to an online solidity event-infants failed to look longer when a moving object appeared to have passed through a wall, compared to when it was stopped by the wall. We speculate on possible reasons for this failure in the General Discussion.

\section{Figure 3}

Infants' looking times in Experiment 1 


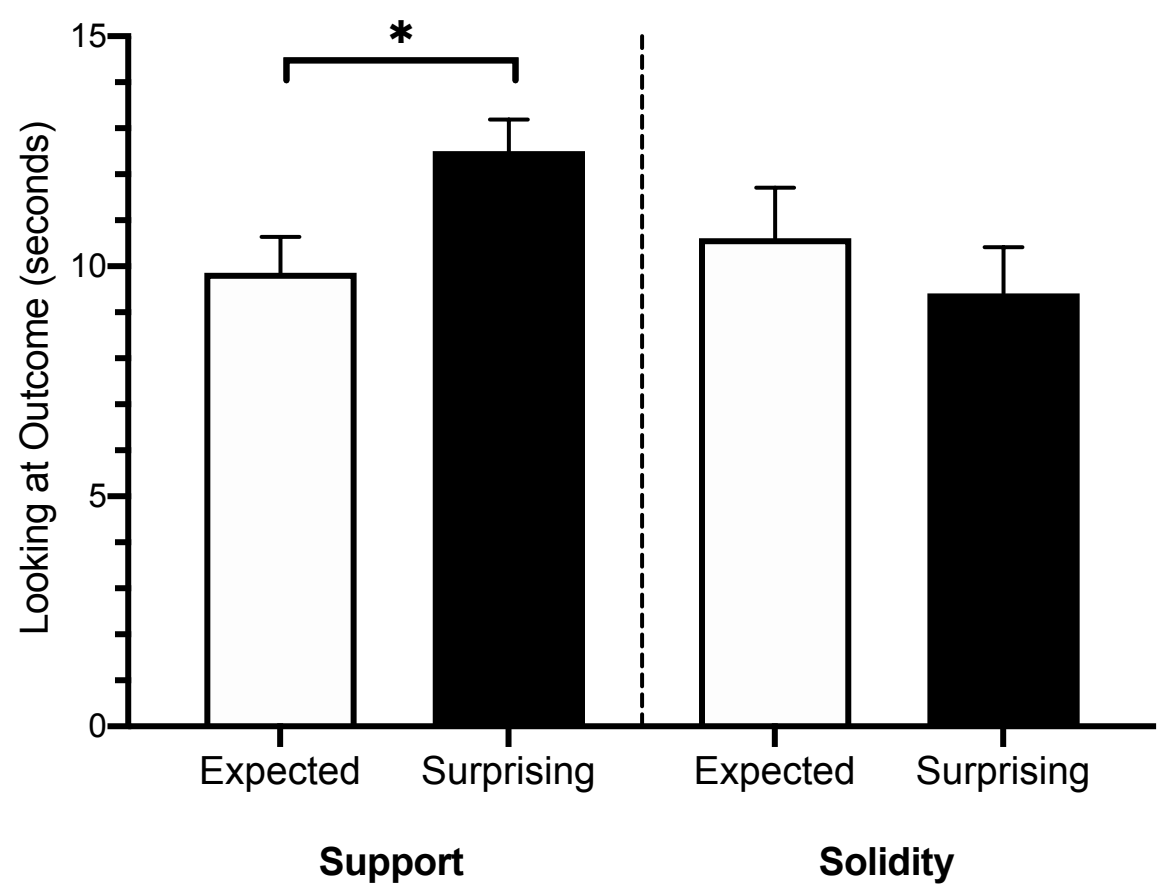

Note. Error bars reflect SEM. * $p=.048$

Experiment 2: Learning following Surprising vs. Expected outcomes of Support and Solidity events

In addition to looking longer at violations-of-expectation, infants and young children also show heightened learning following these surprising events, at least in laboratory settings (Stahl \& Feigenson, 2015; 2017). For example, 11-month old infants saw an object appear to pass through a solid wall, or saw an object appear to violate spatiotemporal continuity by being hidden in Location A but being retrieved at Location B. Immediately following these surprising outcomes, infants were taught new information about the object in the event (that it made a novel sound). Infants learned this new information better, compared to when the same events culminated in expected outcomes (i.e., the object was stopped by the wall, or the object hidden in Location A was retrieved in Location A).

In Experiment 2, we asked whether surprise-induced learning is also observed in online testing. Participants were the same infants from Experiment 1. After their looking at the Surprising or Expected Outcome of the support and solidity events was measured (as reported 
in Experiment 1), we taught infants a novel label for the object that had participated in the event. Then we tested infants' learning of these novel labels.

\section{Method}

\section{Participants}

The same infants from Experiment 1 participated in Experiment 2.

\section{Procedure}

Recall that infants saw two events: one support event and one solidity event (one culminating in a Surprising Outcome and the other in an Expected Outcome). For each of these two events, after infants' looking at the outcome had been measured for 15 seconds, the stage curtain was lowered. Then, after 5 seconds it was raised to reveal the Target Object (i.e., the object that had participated in the preceding support or solidity event) and the Distractor Object (i.e., the object that had been seen during the preceding event, but was an "innocent bystander" rather than the main focus of the event) stationary on the left and right sides of the stage (spatial position counterbalanced across infants) (Figure 4, Panel 1). Infants' looking was measured for 5 seconds- - this provided a baseline index of infants' visual interest in each object. After this, the experimenter reached down, pointed to the Target Object, and said either, "That's a gaffa! Yeah, a gaffa!" (following the Support event) (Figure 4, Panel 2), or "That's a diffy! Yeah, a diffy!" (following the Solidity event), then lowered the curtain.

Finally, infants' learning of the novel label was measured. The curtain was raised to reveal the Target and Distractor objects in the same spatial positions in which they had just been seen. The experimenter, who was not visible to infants, then said, "Where's the [gaffa/ diffy]? Look at the [gaffa/ diffy]!" (Figure 4, Panel 3). Infants' looking was measured for 10 seconds, starting from the onset of the first utterance of the Target Object label. After 10 seconds, the curtain was lowered briefly and raised again to reveal the objects once more, now having switched locations on the stage. Again, infants were prompted to look at the Target Object by the offstage experimenter, as in the previous trial. This resulted in two learning test 
trials per event type (two trials measuring learning following a Surprising outcome and two trials measuring learning following an Expected outcome).

\section{Figure 4}

Schematic of Experiment 2 support event
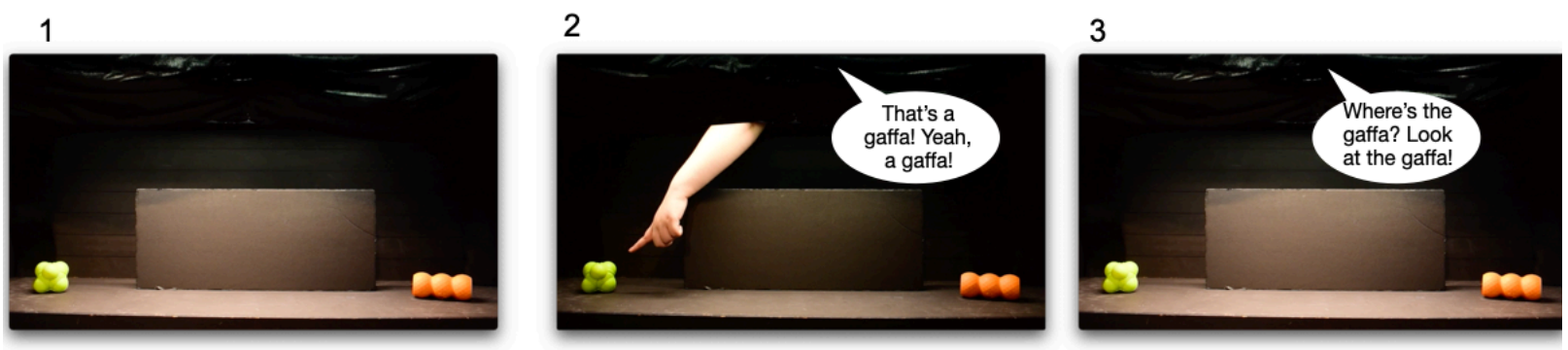

Note. Infants saw the Target and Distractor Objects on either side of the stage for 5 seconds, during which baseline looking to each object was measured (Panel 1). The experimenter then pointed to and labeled the Target Object (Panel 2). Finally, infants were prompted to look at the Target Object using using its novel label, and looking to each object was measured (Panel 3).

\section{Results}

Infants' looking to the Target and Distractor objects during the baseline and test periods was coded offline by two experienced observers. Twenty percent of the video footage was coded by both observers; intercoder reliability was $93.4 \%$. Infants' raw looking times were then converted to learning scores (as in Stahl \& Feigenson, 2015). First, we calculated the proportion of time infants spent looking at the Target Object during the baseline measurement period, by dividing each infant's total looking at the Target Object by their total looking at the Target Object plus their total looking at the Distractor Object. This was done in the same way for each test trial. We then subtracted the proportion of time infants looked at the Target Object during the baseline from the average proportion they looked at it during the two test trials. A positive learning score would indicate an increased tendency to correctly look at the Target Object when hearing it labeled, over and above any baseline preference to look at that object. 
We examined infants' learning scores using a repeated measures ANOVA, with Outcome Type (Surprising vs. Expected) and Trial (first vs. second learning trial) as within subjects factors, and Outcome Event Pairing (whether infants saw a Solidity event with a Surprising Outcome and a Support event with an Expected Outcome, or a Solidity event with an Expected Outcome and a Support event with a Surprising Outcome) and Outcome Order (Surprising Outcome or Expected Outcome first) as between-subject factors. This analysis revealed a significant main effect of Outcome Type, $F(1,20)=7.97, p=.011, \eta^{2}=.29$; infants learned better following a Surprising Outcome $(M=0.09, S D=0.28)$ than an Expected Outcome $(M=-0.12, S D=0.26)$. We also observed a marginally significant main effect of Event Outcome Pairing, $F(1,20)=3.20, p=.089, \eta^{2}=.14$, the difference in infants' learning following Surprising versus Expected Outcomes was greater for infants who had seen the Support event culminate in a Surprising Outcome, compared to infants who saw the Solidity event culminate in a Surprising Outcome. There were no other main effects or interactions.

As in Experiment 1, we conducted planned comparisons to more closely examine infants' performance across the two event types. We found that infants learned the novel label better following a violation of object support $(M=0.13, S D=0.28)$, compared to following a nearly identical event that did not violate object support $(M=-0.22, S D=0.21), t(46)=-2.177, p$ $=.035$ (Figure 5). In contrast, the difference in infants' learning following a violation of object solidity $(M=.05, S D=0.29)$ versus an event that did not violate object solidity $(M=-0.01, S D=$ 0.26), was not significant $t(46)=-1.042, p=.303$ (Figure 5).

Thus, the results of Experiment 2 parallel those of Experiment 1. Most critically, we found that we were able to replicate the effect of surprise-induced learning using online methods: infants learned significantly better following a surprising outcome of an object support event, compared to following an expected outcome. Although there was no significant Outcome Type $x$ Outcome Event Pairing interaction in the ANOVA, simple t-tests suggested that these infants did not learn better following a surprising outcome of an object solidity event. 


\section{Figure 5}

Infants' learning scores in Experiment 2

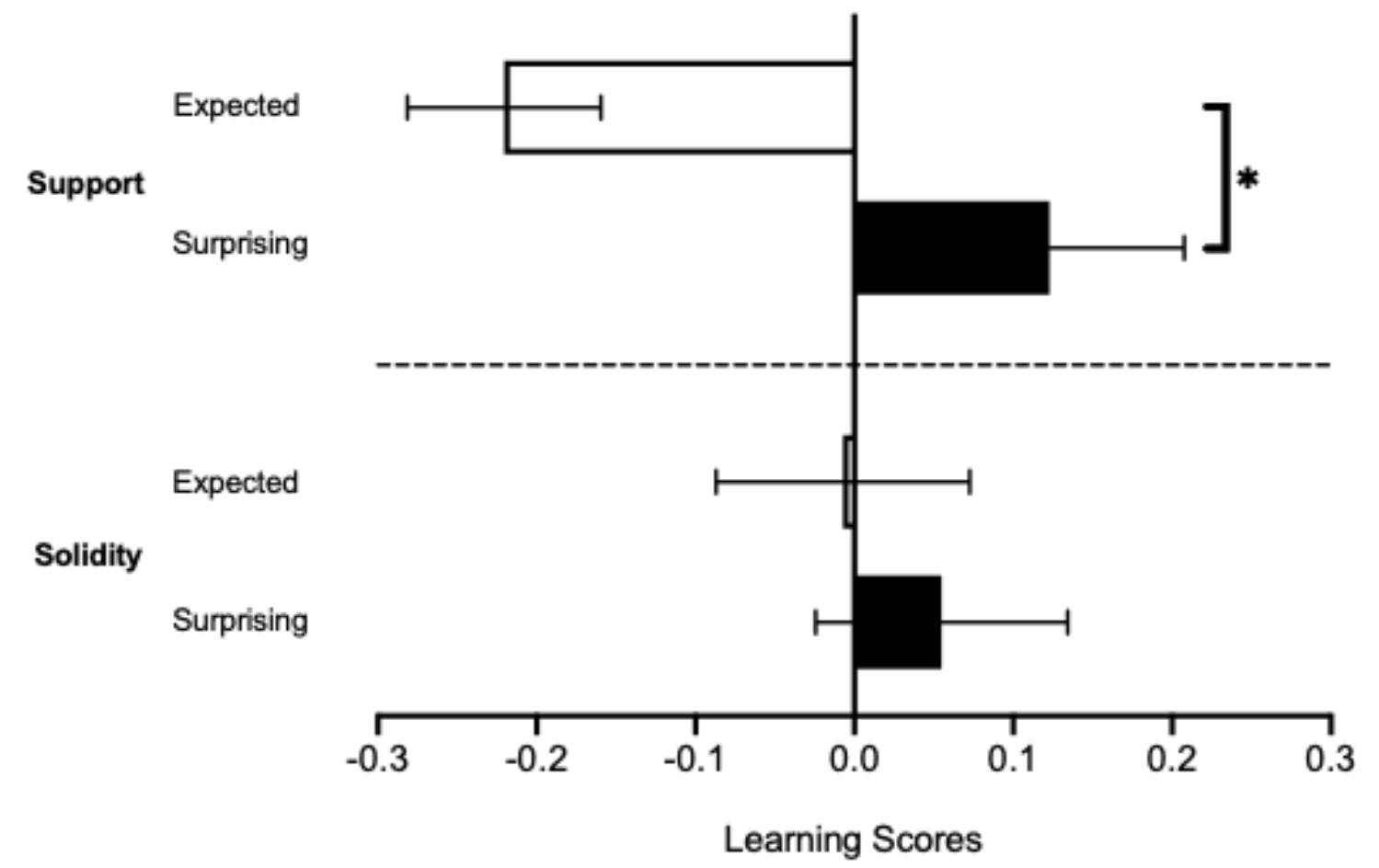

Note. Positive learning scores indicate longer looking at the correct Target Object following object labeling. Error bars reflect SEM. ${ }^{*} p=.035$

\section{Experiment 3: Preferential Looking}

A third classic paradigm in infant cognition research is the paired preference procedure. In this paradigm, infants see two contrasting stimuli while hearing auditory input, and their relative looking at the two images is measured. Experiments using this paradigm find that, for example, infants hearing someone utter a particular vowel sound look longer at images of faces whose lip shape matches rather than mis-matches that vowel sound (Patterson \& Werker, 2003), that infants look longer at a correct than an incorrect referent when hearing an object label (e.g., "Find the shoe"; "Do you see the shoe?”; Golinkoff et al., 1987; Reznick \& Goldfield, 1992; Schafer \& Plunkett, 1998), and look longer at an image of multiple novel objects than an 
image of a single object when hearing a sentence marking plurality ("Look, there are some blickets!" vs. "Look, there is a blicket!"; Kouider, Halberda, Wood, \& Carey, 2006). Although Scott et al. (2017) demonstrated successful looking in an online paired preference task by 24 - to 36-month old children, it is not yet known whether preferential looking can be adapted to online testing with infants, who are too young to receive verbal task instructions. In addition, the success observed by Scott and colleagues involved a large sample of participants $(N=138$, with $\mathrm{N}=67$ in the final analysis). Here we wanted to know whether preferential looking could be obtained online with infants, and with a sample size more typical of in-person laboratory experiments.

In Experiment 3 we asked whether infants tested online could use a person's emotional utterance to determine which of two possible objects was the most likely elicitor. The method was based on a previous in-lab study by Wu et al. (2017), in which infants saw images of two objects that, for adults, were expected to elicit contrasting positive emotional responses (e.g., a dessert and a light up toy-one of which might be perceived as delicious, and the other as exciting or fun). Infants heard an emotion-relevant utterance (e.g., "Yum!"), and their looking was measured. Wu et al. (2017) found that 18- to 23-month-olds looked at the predicted image when they heard a given utterance in a laboratory setting (e.g., infants looked at the dessert after they heard "Yum!"). In our experiment, 16-month-old infants saw an image of a dessert on one side of the screen and an image of a stuffed animal toy on the other, then heard a female speaker either say "Yum!" or the neutral "Oh!”. We hypothesized that infants would look longer at the dessert when they heard "Yum!" but would have no looking preference between the two objects when they heard "Oh!"

\section{Method}

\section{Participants}

Twenty 14- to 18-month-old infants participated (M: 16.46 months, range: 14.04 months to 17.95 months, SD: 1.04 months; 15 girls). Nine infants sat in a highchair and 11 sat in a 
parent's lap. Data from one additional infant was excluded due to low video quality. One infant was identified by their parent as Asian, 18 as White, and one as belonging to more than one race; one infant was also identified as Hispanic/ Latinx. The average screen size across participants was 14.25 inches (range: 13 inches to 20 inches, $S D=2.00$ inches).

\section{Procedure}

Calibration proceeded as in Experiment 1-infants saw a series of animated stimuli appear in the center, left, and right of the screen, accompanied by attention-getting sounds. This served to engage infants with the screen and allowed the coders to see what it looked like when each infant was looking in the locations where the upcoming test stimuli would appear. Following calibration, each infant participated in two test blocks; each block was comprised of one baseline trial and two test trials. On one block infants saw a piece of pink-frosted cake on one side of the screen and a brown teddy bear on the other; on the other block infants saw a brown cupcake on one side and a green toy turtle on the other (Figure 6). If infants saw the dessert on the left for the first block, they saw it on the right for the second block; order of sideobject pairing was counterbalanced across participants.

Baseline Trial. In each baseline event, the two objects appeared simultaneously and a recorded female voice said, "Look at these!" The objects remained visible for eight seconds, during which infants' looking time was recorded. Then the two objects disappeared, and a beach ball appeared and bounced twice in the middle of the screen, to redirect infants' attention back to the center of the screen before the test trials began.

Test Trials. On each test trial, infants saw the same two objects from the preceding baseline trial, in the same locations. After one second, they heard a recorded female voice say either "Yum!" or "Oh!” (Figure 6). Infants' looking was recorded for the subsequent 10 seconds while the objects remained visible onscreen. After 10 seconds, the two objects disappeared and the beach ball appeared in the center of the screen and bounced twice again, to redirect infants' attention. Then infants were presented with the second test trial, which was identical to the first 
except for the utterance. If infants had heard "Yum!" on the first test trial, then they heard "Oh!" on the second, and vice versa.

\section{Figure 6}

\section{Schematic of Experiment 3}

Test Trial 1

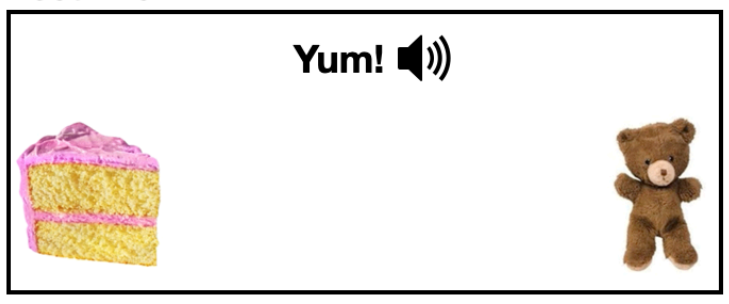

Test Trial 2

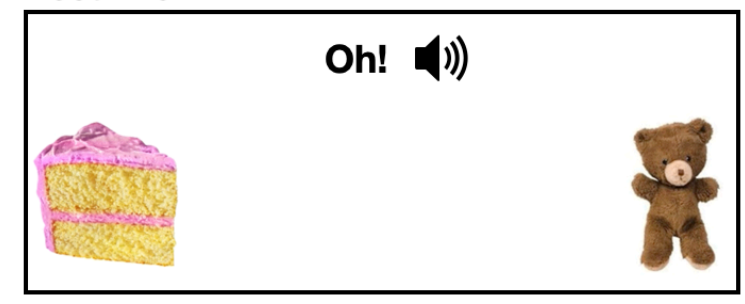

\section{Results}

Infants' looking to each object was coded offline by two experienced observers. Twenty percent of the video footage was coded by both observers; intercoder reliability was $95.5 \%$. Infants' raw looking times were then converted to preference scores: we divided the time each infant spent looking at the dessert on each test trial by their total looking to either object during the test trial. We then subtracted from this the time infants' spent looking at the dessert during the baseline trial divided by their total looking to either object during the Baseline trial. This yielded a measure of increased preference during the test trial. A positive preference score would indicate an increased preference to look at the dessert following the utterance, relative to infants' preference to look at the dessert during baseline.

We analyzed infants' preference scores using a repeated measures ANOVA with Utterance ("Yum!" vs. "Oh!”) and Trial (first vs. second instance of utterance) as within-subject factors, and Block Order (cake slice and teddy bear first vs. cupcake and turtle first) as a between-subjects factor. This revealed a significant main effect of Utterance, $F(1,18)=8.74, p=$ $.008, \eta^{2}=.33$, (Figure 7), with infants showing a greater preference for the dessert after hearing "Yum!" than after hearing "Oh!". As hypothesized, infants also looked longer at the dessert after 
hearing "Yum!" than would be expected by chance, $t(19)=2.46, p=0.024,95 \% \mathrm{Cl}[0.01,0.12]$, whereas when they heard "Oh!" they looked equally at the two stimuli, $t(19)=-1.05, p=0.31$, $95 \% \mathrm{Cl}[-.07,0.02]$. We also observed a main effect of Block Order, $F(1,18)=5.37, p=.03, \eta^{2}=$ 23; infants whose first test block presented the cupcake and the turtle looked longer at the dessert across both blocks $(M=.07, S D=.16)$, compared to infants whose first block presented the cake and the teddy bear $(M=-0.02, S D=0.16)$. No other main effects or interactions were observed.

Infants' longer looking at the dessert after hearing "Yum!" demonstrates that preferential looking can be successfully captured via online testing. Moreover, this evidence corroborates prior work, here demonstrating that younger 16-month-old infants have expectations about the kinds of objects that elicit certain responses. Critically, infants' at-chance looking when hearing the neutral utterance "Oh!" confirms that infants did not simply have an overall preference to look at cakes and cupcakes, relative to non-food items.

\section{Figure 7}

Infants' average preference to look to the dessert image after hearing "Yum!" or "Oh!" in Experiment 3

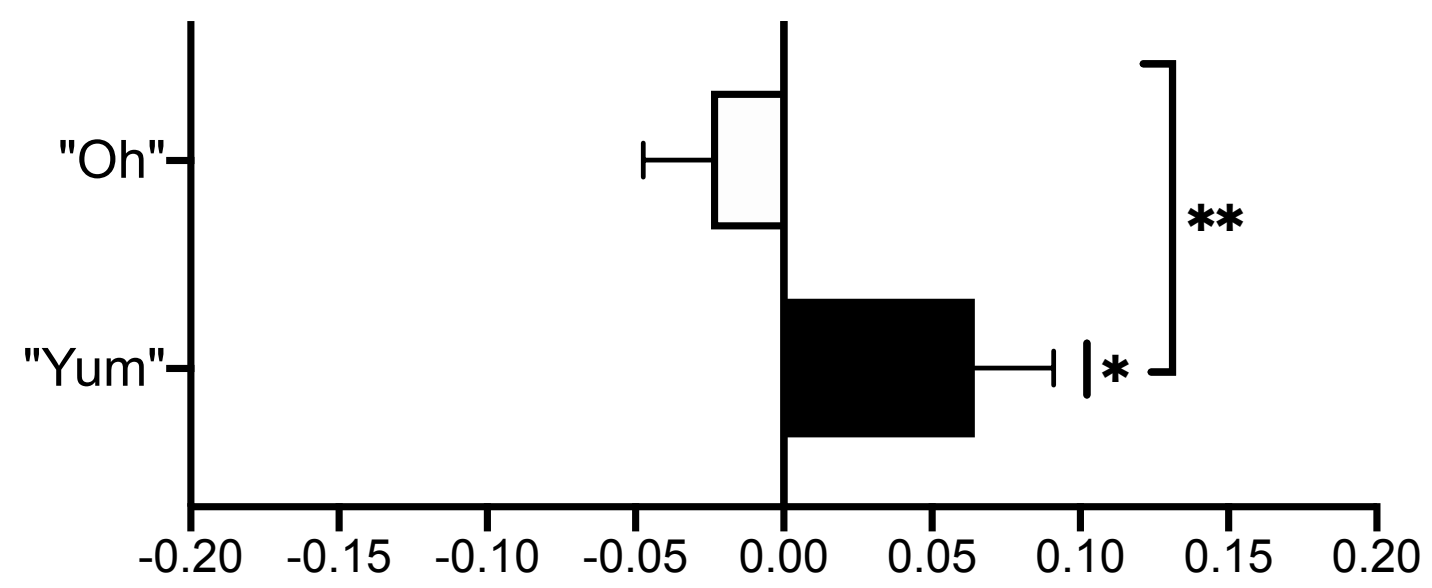

Preference to look at the dessert 
Note. Error bars reflect SEM. ${ }^{*} p=.024,{ }^{* *} p=.008$.

\section{General Discussion}

In three experiments, we asked whether three different infant looking time paradigms could be adapted to an online testing format using experimenter-mediated methods. In Experiment 1 we found that infants looked longer at an event in which the principle of object support was violated, compared to an event that did not violate object support. However, the same infants showed no evidence of detecting a violation of object solidity-a result that contrasts with in-lab studies (e.g., Perez \& Feigenson, 2020; Spelke et al., 1992). In Experiment 2 we found that infants' word learning was enhanced learning following a violation of object support, compared to learning following a nearly identical event that did not culminate in a violation. Finally, in Experiment 3 we found that infants looked longer at images that matched an emotional utterance (i.e., looked longer at a dessert than a toy when hearing "Yum!"). Together, these results suggest that infant looking-time paradigms can be successfully adapted for online testing.

Despite the successful findings reported above, our results also suggest that online testing differs in some important ways from in-lab testing. The failure of infants in Experiment 1 to show increased looking at a solidity violation implicates some important potential differences between our video-recorded stimuli and the live events that we and others have shown infants in the lab. We suspect that one difference between online and in-lab testing has to do with the visual experience of the test stimuli. Due to the screen-size limitations of remote testing, future studies interested in capturing infants' responses to object trajectories (including continuity violations, instances of the tunnel-effect, launching events) may need to think carefully about the visual angle of the stimuli, which can become distorted when seen on a screen. For example, the most common participant screen-size in our experiments ranged from 13- to 15 -inches. In this small space, the size-ratio of the objects in the foreground of the scene (e.g., the target object) to the objects in the background (e.g., the ramp and walls in our solidity study) differed 
significantly from that in our laboratory set-up. The distance traversed by the object in the solidity events of our Experiments 1 and 2 was much smaller than that in the laboratory, in terms of change in visual angle. This much more minimal evidence of the object's trajectory may have been partly responsible for the null results we observed in the solidity condition of our experiments. In comparison, in our support events, the object's trajectory was less crucial to perceiving the event; the object's lateral movement through space was not required to convey the sense of support vs. lack of support from below.

In addition, future online infant studies may wish to explore the use of infant controlled looking, as opposed to the fixed trial durations that we used, and that were also used by Scott et al. (2017). Infant controlled looking allows trial lengths to vary based on individual infants' behavior; this may provide a more sensitive measure. Our method of using Zoom with a live experimenter could be easily adapted for this-for example, researchers can use slides.com or similar platforms to control stimulus presentation dynamically in real-time (see Supplementary Materials for instructions).

Another motivation of our study was to determine whether experimenter-mediated studies result in lower exclusion rates than asynchronous methods. Experiment 1 had a final sample of 24 infants, but tested a total of 25 additional infants, resulting in a $51 \%$ exclusion rate. However, only 7 (13.5\%) infants were excluded due to online data collection reasons specifically (e.g., video quality and screen size constraints), while the other 18 infants were excluded for reasons similar to in-laboratory experiments (e.g., fussiness, parental interference). Experiment 3 had a much lower total exclusion rate, with only one of 21 infants excluded for low video quality $(4.7 \%)$. Compared to asynchronous testing methods, testing with a live experimenter does seem to reduce rates of participant exclusion. We encourage researchers to consider their study design when deciding between asynchronous and synchronous infant testing platforms.

Based on our experience testing infants over the past year, online infant experiments seem a viable way to make continued research progress even when in-person laboratory testing 
is not possible. Furthermore, online testing need not be a replacement for in-lab testing; it can function in parallel, as an additional means to reach families who lack the means to travel to a campus laboratory, or whose schedules make laboratory visits inconvenient. Researchers who wish to recruit special populations of infants, or to test infants from racial, ethnic, or socioeconomic backgrounds that differ from the population geographically proximal to their lab also may wish to explore this testing format. Of course, whether online testing diversifies an experiment sample depends on the individual circumstances of the lab, and on the population they would otherwise have tested. It also is important to note that participants from families with access to home computers and reliable internet, and with an interest in volunteering for experiments, are more likely to be White and from middle or upper-middle socioeconomic backgrounds (Lourenco \& Tasimi, 2020). As such, the question of how research findings generalize outside the context of the experimental sample persists for online experiments, just as it long has for those conducted in the laboratory.

In summary, we see this as an exciting time for infant research. Our field is poised to embrace ambitious endeavors at scales that were unthinkable in the earlier decades of cognitive development research. Multi-lab replication efforts like ManyBabies (Byers-Heinlein et al., 2020; Byers-Heinlein et al., 2021), other types of large-scale collaborative networks (Sheskin et al., 2020), and online testing like that pioneered by Looklt (Scott \& Schulz, 2017; Scott et al., 2017) and extended in the present work all have the potential to broaden the scope and impact of research on the developing mind. We hope our work can contribute to that effort. 


\section{References}

Aslin, R.N., (2007). What's in a look?. Developmental Science, 10(1), 48-53. 10.1111/j.14677687.2007.00563.x

Baron-Cohen, S., Leslie, A. M., \& Frith, U. (1985). Does the autistic child have a "theory of mind"?. Cognition, 21(1), 37-46. 10.1016/0010-0277(85)90022-8

Byers-Heinlein, K., Bergmann, C., Davies, C., Frank, M. C., Hamlin, J. K., Kline, M., Kominsky, J. F., Kosie, J. E., Lew-Williams, C., Liu, L., Mastroberardino, M., Singh, L., Waddell, C. P. G., Zettersten, M., \& Soderstrom, M. (2020). Building a collaborative psychological science: Lessons learned from ManyBabies 1. Canadian Psychology/Psychologie canadienne, 61(4), 349-363.10.1037/cap0000216

Byers-Heinlein, K., Tsui, A. S. M., Bergmann, C., Black, A. K., Brown, A., Carbajal, M. J., Durrant, S., Fennell, C. T., Fiévet, A.-C., Frank, M. C., Gampe, A., Gervain, J., Gonzalez-Gomez, N., Hamlin, J. K., Havron, N., Hernik, M., Kerr, S., Killam, H., Klassen, K., ... Wermelinger, S. (2021). A Multilab Study of Bilingual Infants: Exploring the Preference for Infant-Directed Speech. Advances in Methods and Practices in Psychological Science. 10.1177/2515245920974622

Golinkoff, R. M., Hirsh-Pasek, K., Cauley, K. M., \& Gordon, L. (1987). The eyes have it: Lexical and syntactic comprehension in a new paradigm. Journal of Child Language, 14(1), 2345. $10.1017 / \mathrm{S} 030500090001271 \mathrm{X}$

Hood, B. M. (1995). Gravity rules for 2-to 4-year olds?. Cognitive Development, 10(4), 577-598. 10.1016/0885-2014(95)90027-6

Kouider, S., Halberda, J., Wood, J., \& Carey, S. (2006). Acquisition of English number marking: the singular-plural distinction. Language Learning and Development, 2(1), 1-25. 10.1207/s15473341Ild0201_1

Lourenco, S.F. \& Tasimi, A. (2020). No participant left behind: conducting science during COVID-19. Trends in Cognitive Sciences, 24(8), 583-584. 10.1016/j.tics.2020.05.003 
Needham, A. \& Baillargeon, R. (1993). Intuitions about object support in 4.5-month-old infants. Cognition, 47(2), 121-148. 10.1016/0010-0277(93)90002-D

Patterson, M.L. \& Werker, J.F. (2003). Two-month-old infants match phonetic information in lips and voice. Developmental Science, 6(2), 191-196. 10.1111/1467-7687.00271

Perez, J., \& Feigenson, L. (2020). Violations of expectation trigger infants to search for explanations. PsyArXiv. 10.31234/osf.io/eahjd

Reznick, J. S., \& Goldfield, B. A. (1992). Rapid change in lexical development in comprehension and production. Developmental Psychology, 28(3), 406. 10.1037/0012-1649.28.3.406

Rhodes, M., Leslie, S. J., \& Tworek, C. M. (2012). Cultural transmission of social essentialism. Proceedings of the National Academy of Sciences, 109(34), 13526-13531. 10.1073/pnas.1208951109

Rhodes, M., Rizzo, M.T., Foster-Hanson, E., Moty, K., Leshin, R.A., Wang, M., Benitez, J., \& Ocampo, J.D. (2020). Advancing developmental science via unmoderated remote research with children. Journal of Cognition and Development, 21(4), 477-493. $10.1080 / 15248372.2020 .1797751$

Schafer, G., \& Plunkett, K. (1998). Rapid word learning by fifteen-month-olds under tightly controlled conditions. Child development, 69(2), 309-320. 10.2307/1132166

Scott, K., Chu, J., \& Schulz, L. (2017). Lookit (Part 2): Assessing the viability of online developmental research, results from three case studies. Open Mind, 1(1), 15-29. 10.1162/OPMI_a_00001

Scott, K., \& Schulz, L. (2017). Lookit (Part 1): A new online platform for developmental research. Open Mind, 1(1), 4-14. 10.1162/OPMI_a_00002

Sheskin, M. \& Keil, F. C. (2018). TheChildLab.com: A video chat platform for developmental research. PsyArXiv. 10.31234/osf.io/rn7w5

Sheskin, M., Nadal, A., Croom, A., Mayer, T., Nissel, J., \& Bloom, P. (2016). Some equalities 
are more equal than others: quality equality emerges later than numerical equality. Child Development, 87(5), 1520-1528. 10.1111/cdev.12544

Sheskin, M., Scott, K., Mills, C.M., Bergelson, E., Bonawitz, E., Spelke, E.S., Fei-Fei, L., Keil, F.C., Gweon, H., Tenenbaum, J.B., Jara-Ettinger, J., Adolph, K.E., Rhodes, M., Frank, M.C., Mehr, S.A., \& Schulz, L. (2020). Online developmental science to foster innovation access, and impact. Trends in Cognitive Sciences, 24(9), 675-678.

10.1016/j.tics.2020.06.004

Spelke, E.S., Breinlinger, K., Macomber, J., \& Jacobson, K. (1992). Origins of knowledge. Psychological Review, 99(4), 605. doi.org/10.1037/0033-295X.99.4.605

Spelke, E. S., \& Kinzler, K. D. (2007). Core knowledge. Developmental Science, 10(1), 89-96. 10.1111/j.1467-7687.2007.00569.x

Stahl, A. E., \& Feigenson, L. (2015). Observing the unexpected enhances infants' learning and exploration. Science, 348(6230), 91-94. 10.1126/science.aaa3799

Stahl, A. E., \& Feigenson, L. (2017). Expectancy violations promote learning in young children. Cognition, 163, 1-14. 10.1016/j.cognition.2017.02.008

Téglás, E., Vul, E., Girotto, V., Gonzalez, M., Tenenbaum, J. B., \& Bonatti, L. L. (2011). Pure reasoning in 12-month-old infants as probabilistic inference. Science, 332(6033), 10541059. $10.1126 /$ science. 1196404

Tran, M., Cabral, L., Patel, R., \& Cusack, R. (2017). Online recruitment and testing of infants with Mechanical Turk. Journal of Experimental Child Psychology, 156, 168-178. 10.1016/j.jecp.2016.12.003

Wu, Y., Muentener, P., \& Schulz, L. E. (2017). One-to four-year-olds connect diverse positive emotional vocalizations to their probable causes. Proceedings of the National Academy of Sciences, 114(45), 11896-11901. 10.1073/pnas.1707715114

Yuan, S., \& Fisher, C. (2009). "Really? She blicked the baby?" Two-year-olds learn 
combinatorial facts about verbs by listening. Psychological Science, 20(5), 619-626.

10.1111/j.1467-9280.2009.02341.x 
Supplementary Materials for

\section{Online measures of looking and learning in infancy}

Alexis S. Smith-Flores*, Jasmin Perez, Michelle H. Zhang, and Lisa Feigenson

This PDF file includes:

Instructions for Zoom Setup for Stimuli on Computer

Instructions for Zoom Setup for Stimuli on slides.com

Correspondence should be addressed to:

Alexis S. Smith-Flores

alexis-smith@ucsd.edu 


\section{Overview}

For online testing over Zoom, video stimuli can be presented in two ways. The first simply involves the experimenter sharing their screen with a participant. The second involves uploading stimuli to a third- party hosting website (e.g., slides.com), and having the participant view stimuli through that platform. In cases where experiments involve high-resolution stimuli and there are concerns about stimulus quality over Zoom, the third-party hosting platform may be the best solution. We recommend slides.com for this method. This supplementary guide details the set-up and configuration of both of these methods.

\section{Zoom Setup for Stimuli played from Experimenter's Computer}

\section{Recording Setup}

These recording set-up instructions show you how to configure Zoom to record both your screen-shared video stimuli and the video of the participant. This section will also describe how to set the location to which your Zoom recordings will be saved.

1. Open Zoom settings. If using a Mac, click the zoom.us button on the top of the right screen and select Preferences. A new window will appear (Figure 1).

2. Go to Recording, located on the left side of the window.

3. Beside the text that reads Local Recording: Store my recordings at: is the folder that has been selected as the default location for the Zoom recording. To change this, click on whichever folder appears as the default.

4. When you have done this, a drop-down menu will appear and give you the option to Choose a new location.

5. When you have selected a new location for your Zoom recordings (such as your Desktop or your lab's secure video server), click Choose to save your setting. Zoom is now set up to save the recordings to the folder you have selected.

6. To record Zoom participant videos while you are sharing your screen, ensure that the option labeled Record video during screen sharing has a checkmark beside it. If it does not, click the box beside the option. If you do not click this option, you will only record your stimuli, rather than both your stimuli and the participant.

7. To prevent Zoom participant videos from being placed on top of the stimuli in your recording, ensure that the option labeled Place video next to the shared screen 
in the recording has a checkmark beside it. You can see the location of these options in Figure 1.

\section{Figure 1}

\section{Zoom Recording Settings}

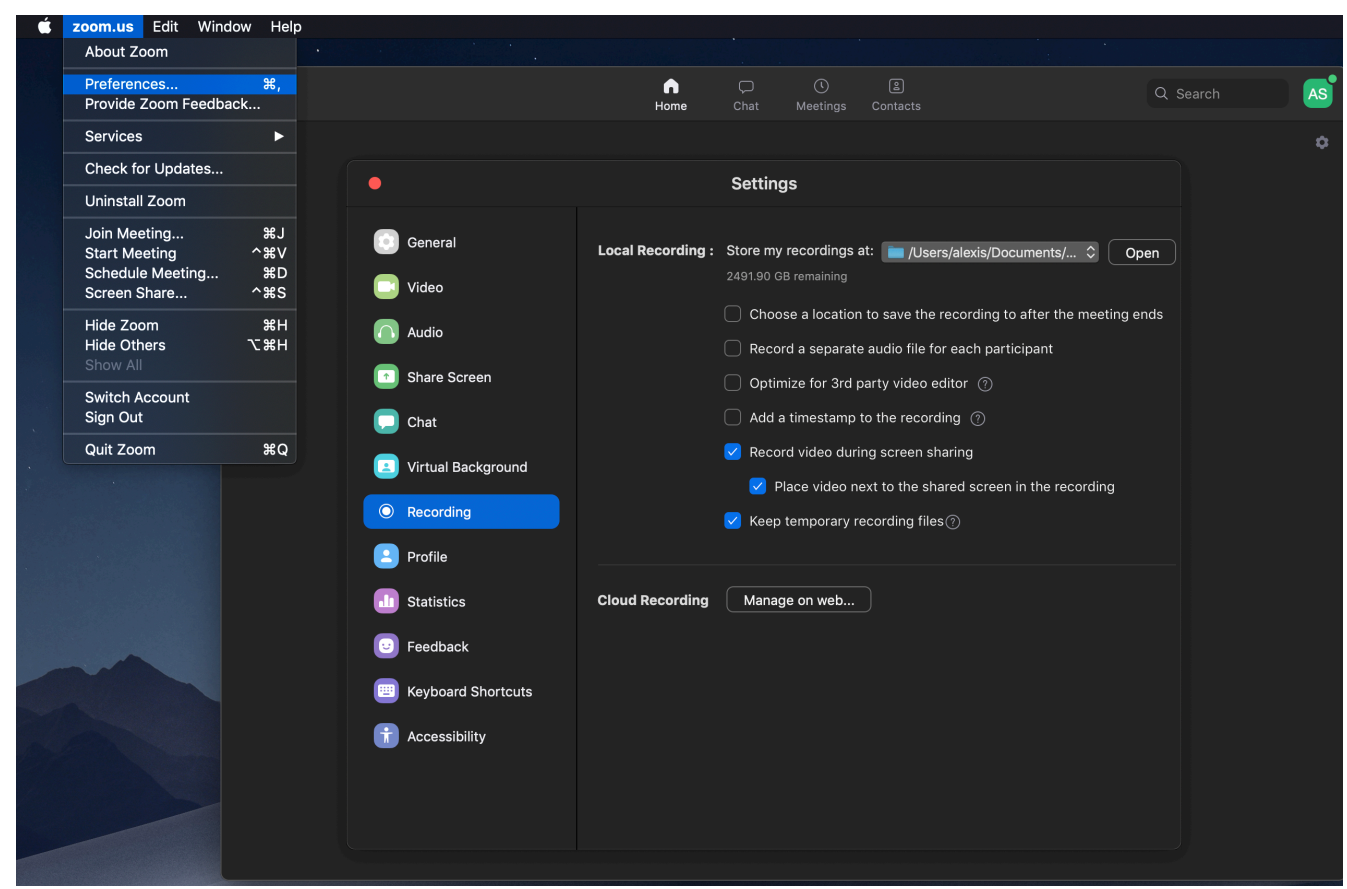

\section{Zoom Call Set Up}

In this section, we review how to configure a Zoom call for an experiment in which the experimenter is screen sharing video stimuli with a participant. This set-up requires using a Zoom feature called Spotlight Video. Spotlight Video enables the experimenter to record only the video being screen shared and the participant's video, without interference from any other videos on the call. In contrast, the typical Zoom recording without the use of Spotlight Video will record whoever is the Active Speaker (whoever is talking or sharing their screen on Zoom). Without Spotlight Video, the experimenter will only record an image of themselves and the stimuli. Importantly, this feature requires that three participants be on the same Zoom call. Below we suggest a work-around in the event that a second live second experimenter is not available.

1. Open the Zoom link you sent to the participant's parent when they scheduled the appointment.

2. Open the video you will be showing to your participant. 


\section{Figure 2}

Example of successful recording setup, displaying stimuli and participant's face

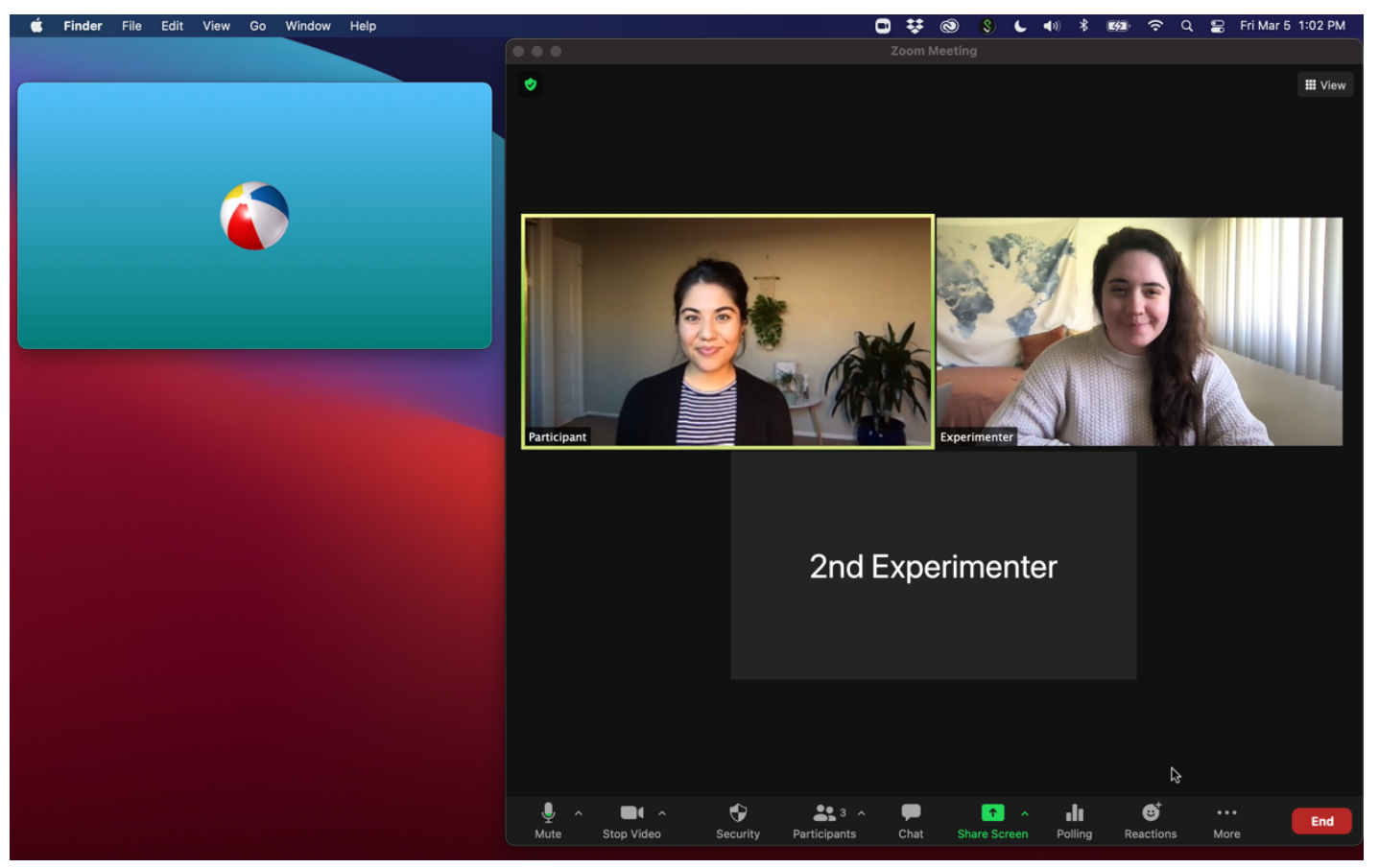

3. Once three people have joined the Zoom call (you, the participant's parent, and the second experimenter) and your Zoom call resembles that in Figure 2, you can enable Spotlight Video.

- If you do not have a second experimenter, you can log onto the Zoom call from an additional device (e.g., smartphone, tablet) using a secondary Zoom account. When you join the call from your second device, ignore any requests to turn on audio or video on your second device.

4. As mentioned at the start of this section, to record the participant without any interference from another participant on the call, you must enable Spotlight Video. Hover over the participant's video and click the three dots that appear, then select Spotlight for Everyone (Figure 3).

\section{Figure 3}

Spotlight the participant's video in order to record their video without interruption from another Zoom participant 


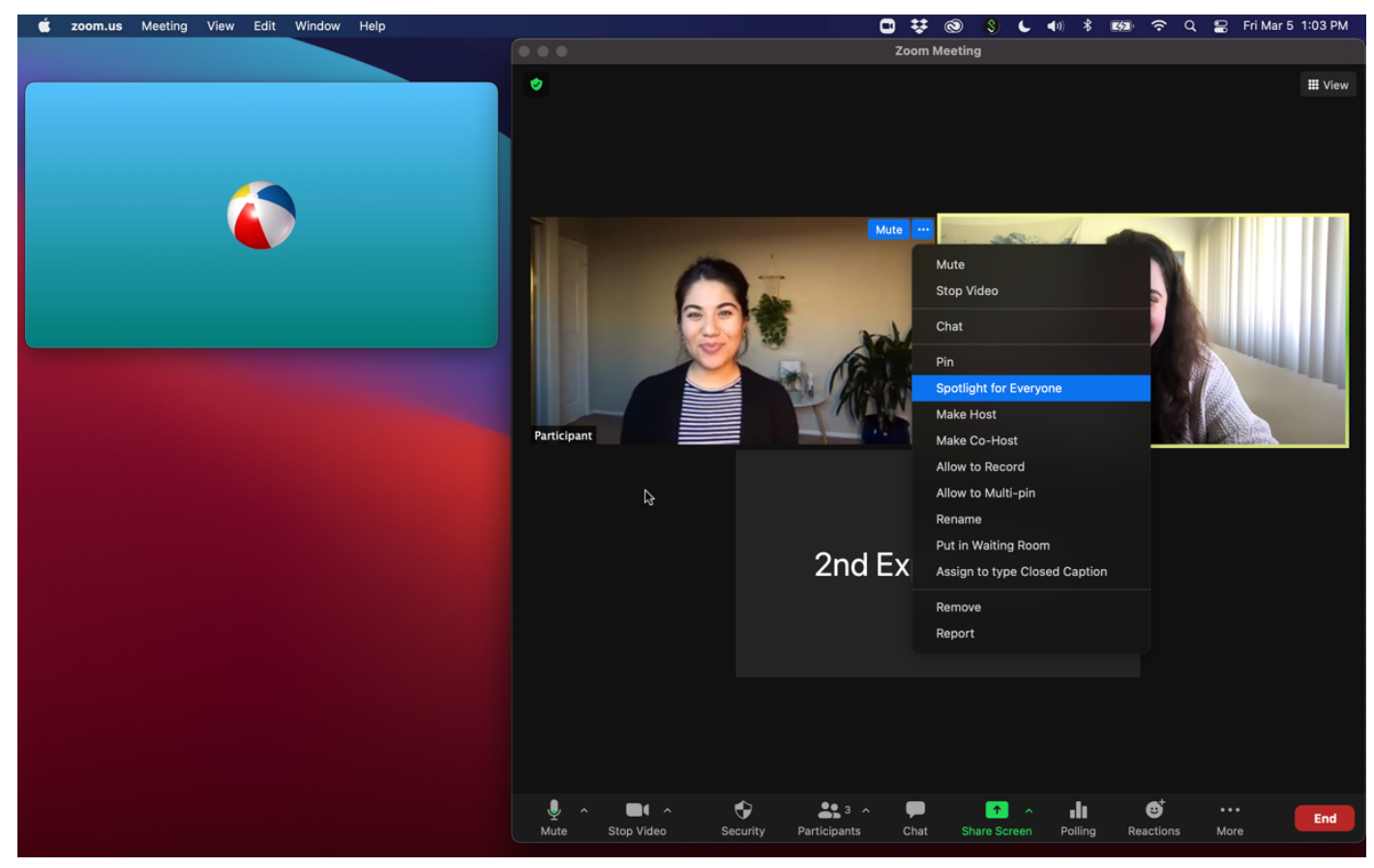

5. Once you have selected Spotlight for Everyone, a notification will appear in the top left of the participant's video that reads, Remove Spotlight. If you see this notification, you have correctly enabled Spotlight Video.

6. When the parent and participant are in position for your experiment (e.g., in highchair, correct distance from their screen) and are ready to begin, click the green Screen Share button at the bottom of your Zoom window.

7. A new Zoom window will appear, allowing you to choose which window from your computer you wish to share. Select the window displaying your stimuli; also select Share sound (Figure 4). When both the video and Share sound have been selected, click the blue Share button in the lower right-hand corner. Your screen will now resemble that in Figure 5. 


\section{Figure 4}

Select your stimuli and Share computer sound, then select Share

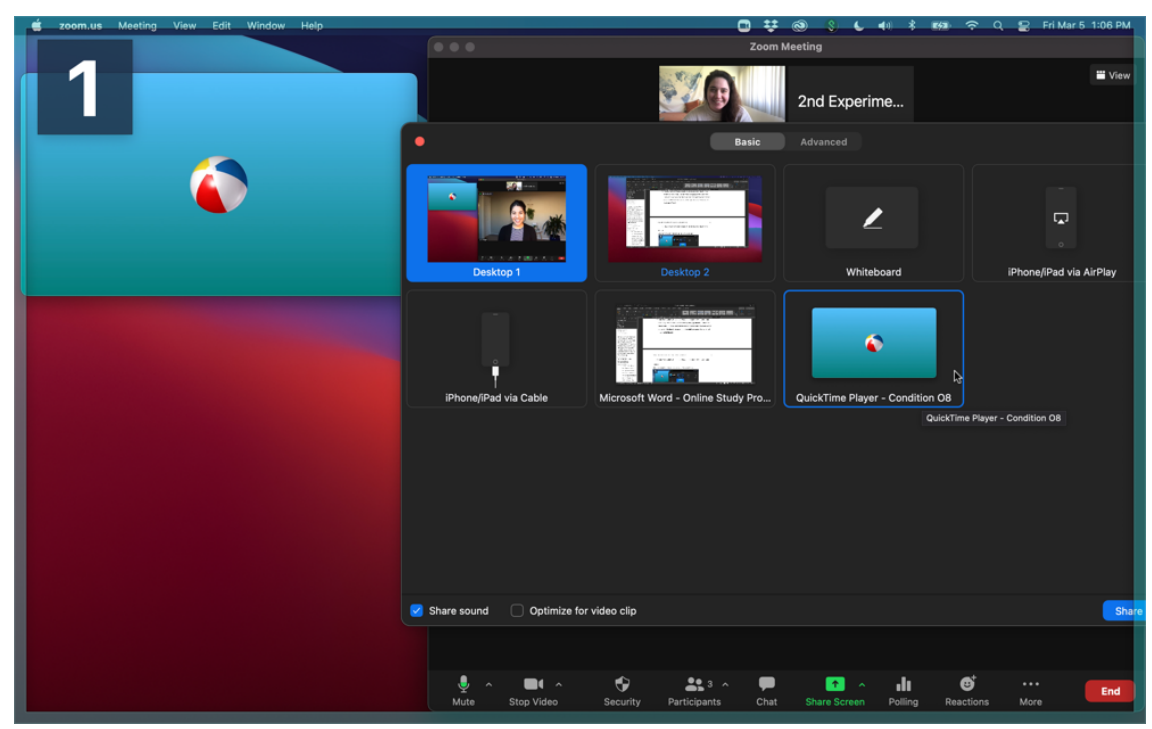

8. On the video panel displaying the video of you and of the participant, select the square button in the middle so that you see only the participant's video (rather than all of the Zoom participant videos). If you do not select this button, Zoom will record everyone's video in very small thumbnails, which will make offline coding of these videos difficult. Selecting the square button will also allow you to enlarge the video of the participant, which will carry over to the Zoom recording (Figure 6).

9. Ask the participant's parent if the shared video appears as full-screen on their computer. Zoom should do this automatically, unless the parent has previously altered their settings.

\section{Figure 5}

Example of successfully screen sharing your stimuli. 


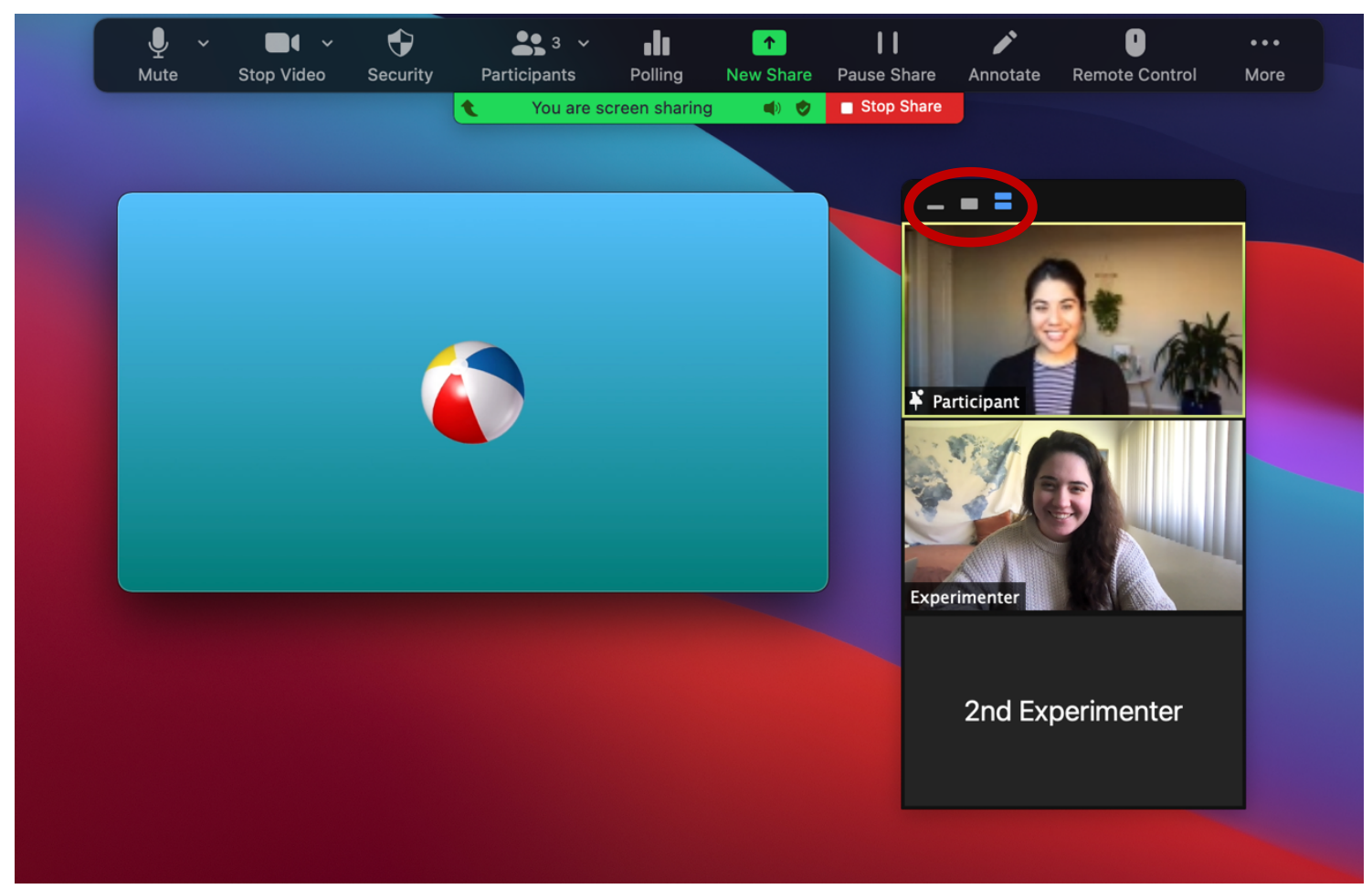

\section{Figure 6}

Switch to single video view by selecting the square on the video panel. Resize participant video to the largest possible size by dragging the bottom left corner of the video outward.

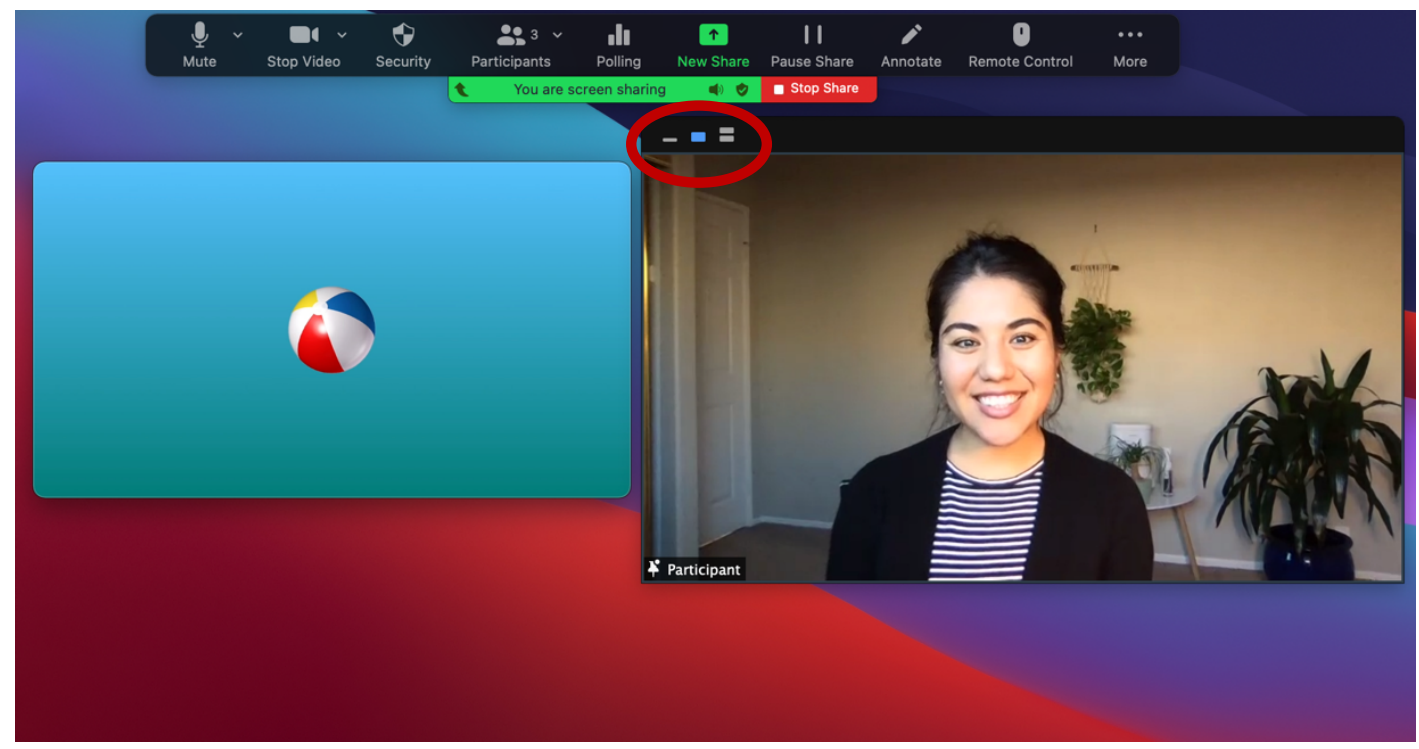

10. In most cases, you will want to have the participant's parent hide the video panel containing everyone's video, so that the child is not looking at an image of themselves or of the experimenter during the testing session. To hide the video 
panel on the participant's screen, ask the parent to move their mouse to the top of their screen, hover over the edge of the zoom window, and click View Options (if this option is not already visible).

11. Ask the parent to click Hide Video Panel

- If Hide Video Panel does not appear as an option, ask them whether Sideby-Side mode is selected. De-selecting Side-by-Side mode should enable the Hide Video Panel feature.

12. Zoom setup is now complete and you can start recording.

13. Click the three dots on the top of your screen that say More. Then click Record on this computer (Figure 7). A red light should appear on the green bar at the top of your screen.

\section{Figure 7}

Record by clicking the three buttons above More, and then select Record on this

Computer.

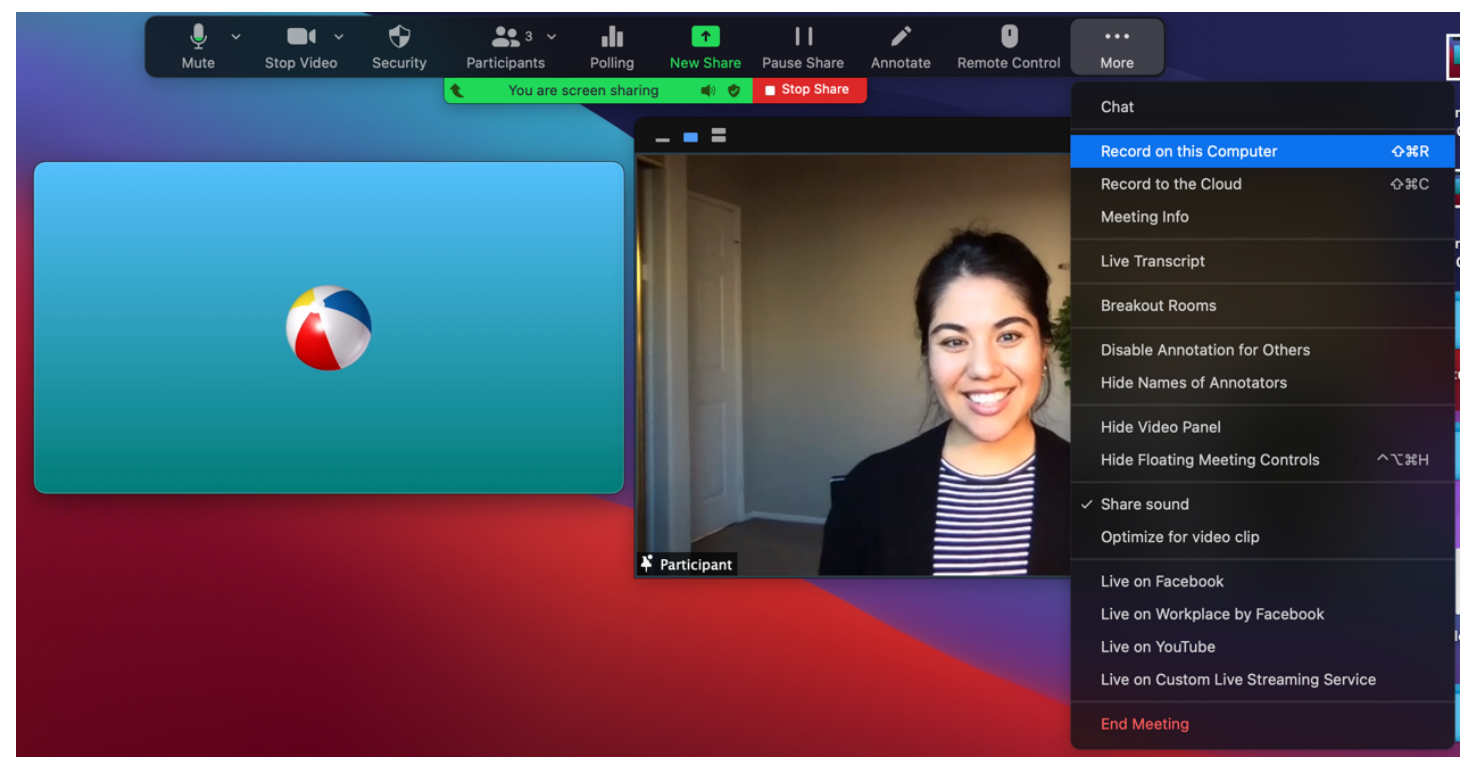

○ Remember to mute yourself before initiating your stimulus presentation so that the participant cannot hear ambient sounds from your testing location.

- If the video of the participant is distorted, you can stop the Zoom video of your camera (stopping your video will minimize interference and should increase the quality of the participant's video). Do not stop your screen share. You can stop your Zoom video by selecting the Stop Video button in the Zoom toolbar at the top of your screen. You can see this button in Figure 7; it is the button second from the left on the toolbar at the top. 
14. When you are ready to end the testing session, click Stop Share (the red button under your Zoom toolbar at the top of your screen) to end the stimulus presentation. You will receive a notification that your video will be converted when the meeting ends.

- Zoom does not allow you to record twice in the same session. If you plan to test sibling participants, you must either pause the recording between participants, or save the recording, end the call, and begin a new call for the next participant.

15. When you end the call, file conversion should begin automatically (Figure 8).

\section{Figure 8}

Zoom automatically converts the recording after meeting ends.

\section{Converting meeting recording}

You have a recording that needs to be converted before viewing

$77 \%$

Stop Converting 


\section{Zoom Setup for Stimuli played on slides.com}

In this section we offer instructions for running an experiment online using slides.com (Slides) and Zoom in tandem. This is a helpful option when there are concerns about the video quality of the stimuli. For stimuli that involve more subtle manipulations (e.g., facial expressions, small perceptual changes) that require higher visual resolution, we recommend using Slides.

\section{Recording Setup}

These instructions show you how to configure Zoom to record both your screenshared video stimuli and the video of the participant. This section will also describe how to set the location to which your Zoom recordings will be saved.

8. Open Zoom settings. If using a Mac, click the zoom.us button on the top of the right screen and select Preferences, a new window will appear (Figure 9).

9. Go to Recording, located on the left side of the window.

10. Beside the text that reads Local Recording: Store my recordings at: is the folder that has been selected as the default location for the Zoom recordings. To change this, click on whichever folder has been chosen as the default.

11. When you have done this, a drop-down menu will appear and give you the option to Choose a new location.

12. When you have selected a new location for your Zoom recordings, such as your desktop or your lab's secure video server, click Choose to save your setting. Zoom is now set up to save the recordings to the folder you have selected.

13. To record Zoom participant videos in the Zoom recording when a screen is being shared, ensure the option labeled Record video during screen sharing has a checkmark beside it. If it does not, click the box beside the option. If you do not click this option, you will only record your stimuli, rather than both your stimuli and the participant.

14. To prevent Zoom participant videos from being on top of the stimuli in your recording, ensure that the option labeled Place video next to the shared screen in the recording has a checkmark beside it. You can see the location of these options in Figure 9.

\section{Figure 9}




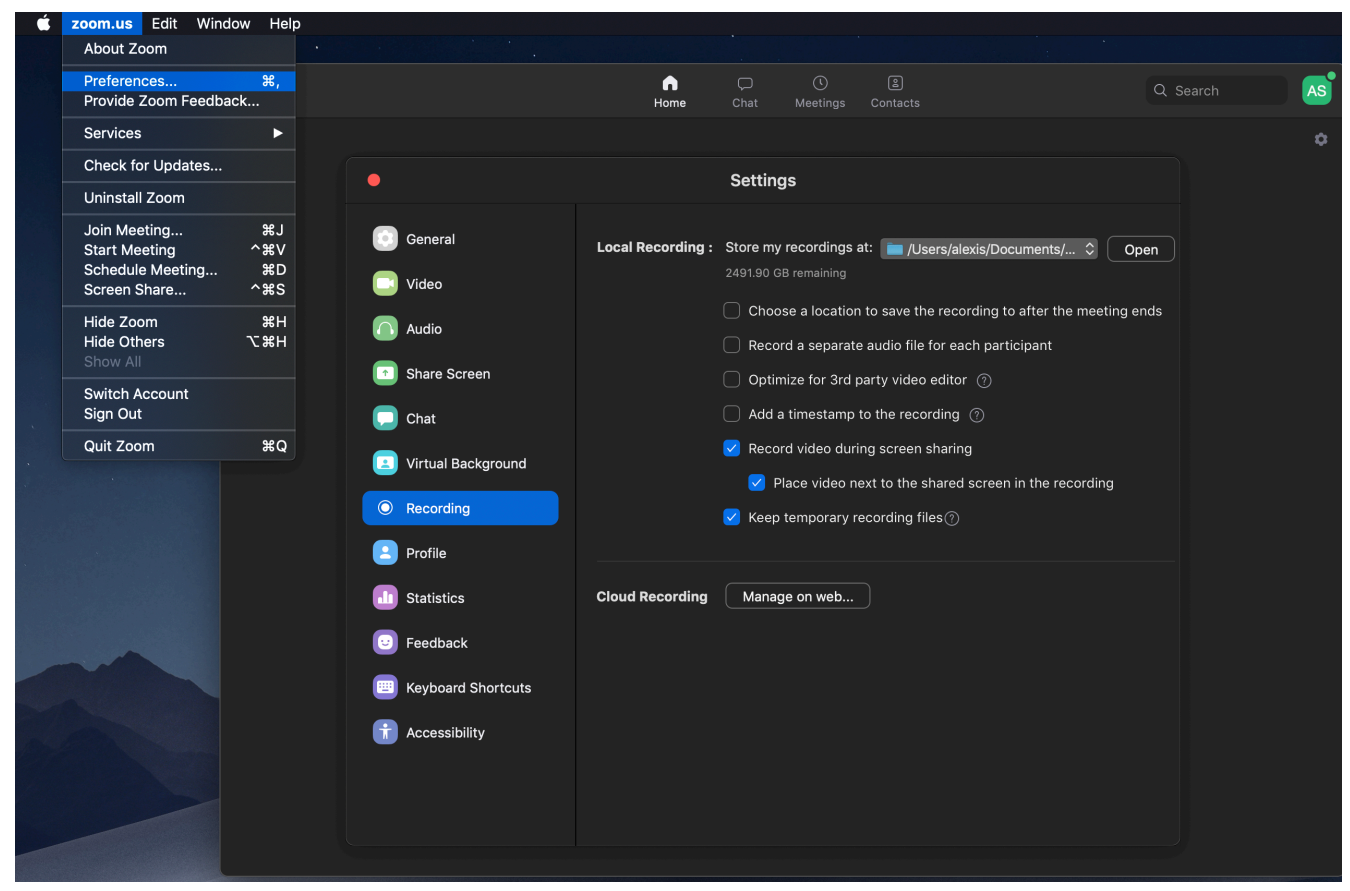

\section{Slides Setup}

1. You must first decide what kind of experiment you are running and what materials you want to upload to Slides. Will you have all your stimuli in a single video that Slides will play without stopping? Will you have multiple videos that you will advance based on infants' looking times (e.g., Is this an infant controlled study?)?

2. Create a slide deck on Slides. Add slides to the deck based on the number of videos you will show the participant. For example, if you have a single video that contains all of the trials, with fixed trial durations, you will only need one slide. If your trials are contingent on each infant's looking, and you have, for example, two familiarization trials $(F)$ and two test trials $(T)$ with separate attention-getter $(A G)$ videos in between each trial (e.g., F-AG-F-AG-T-AG-T), then you will need to create 7 slides.

3. Using Slides is similar to using Microsoft PowerPoint or Apple Keynote. You can upload all your videos to your media library and then add a video to each slide. Once you have added a video to a slide you can change the video's settings. For example, you can have a video play repeatedly by enabling the loop function (Figure 10).

4. You can preview your slide deck by clicking the Play button, located on the left side of the screen under the checkmark.

\section{Figure 10}

Slides video settings 


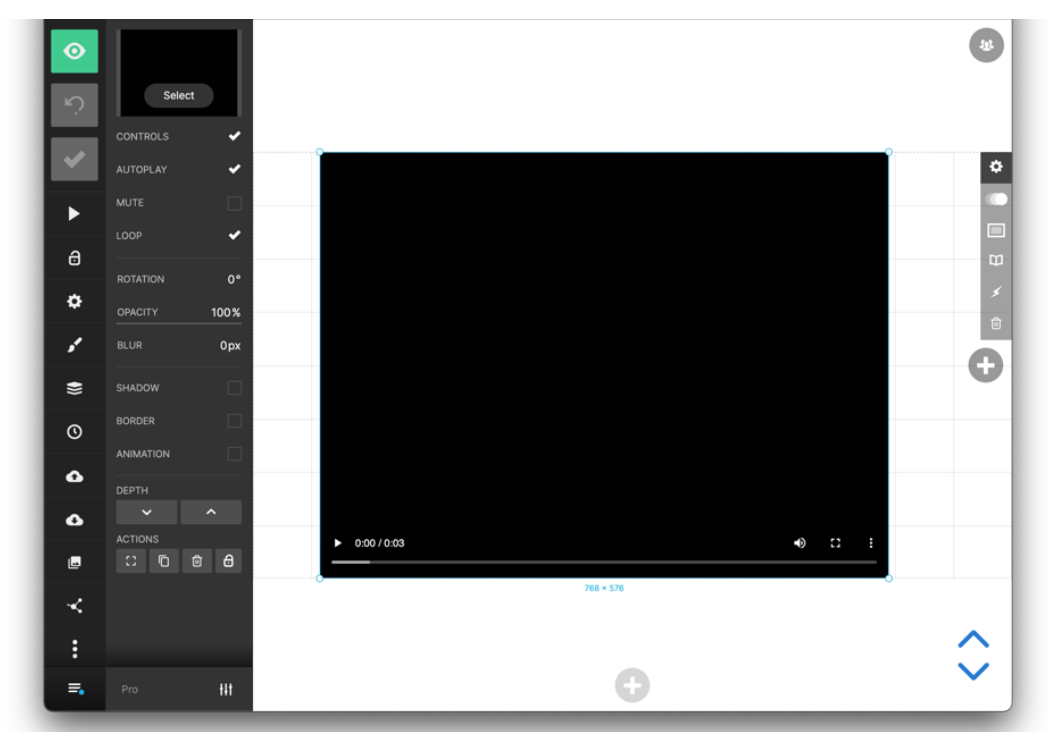

\section{Zoom Call Setup}

In this section, we review how to configure your Zoom call for an experiment in which the experimenter is presenting stimuli using Slides. In this case, the participant's parent must share their screen with the experimenter so that the stimuli being presented are included in the Zoom recording. We recommend using Google Chrome, as this allows the experimenter to mute their Slides window and thereby avoid any echo or other audio feedback in the Zoom recording.

1. Open the Zoom link you sent to the participant's parent when they scheduled the appointment.

2. Open Slides in Google Chrome. Once you have Slides open, click on the slide deck you created and select Present (Figure 11). 


\section{Figure 11}

Opening your slide deck

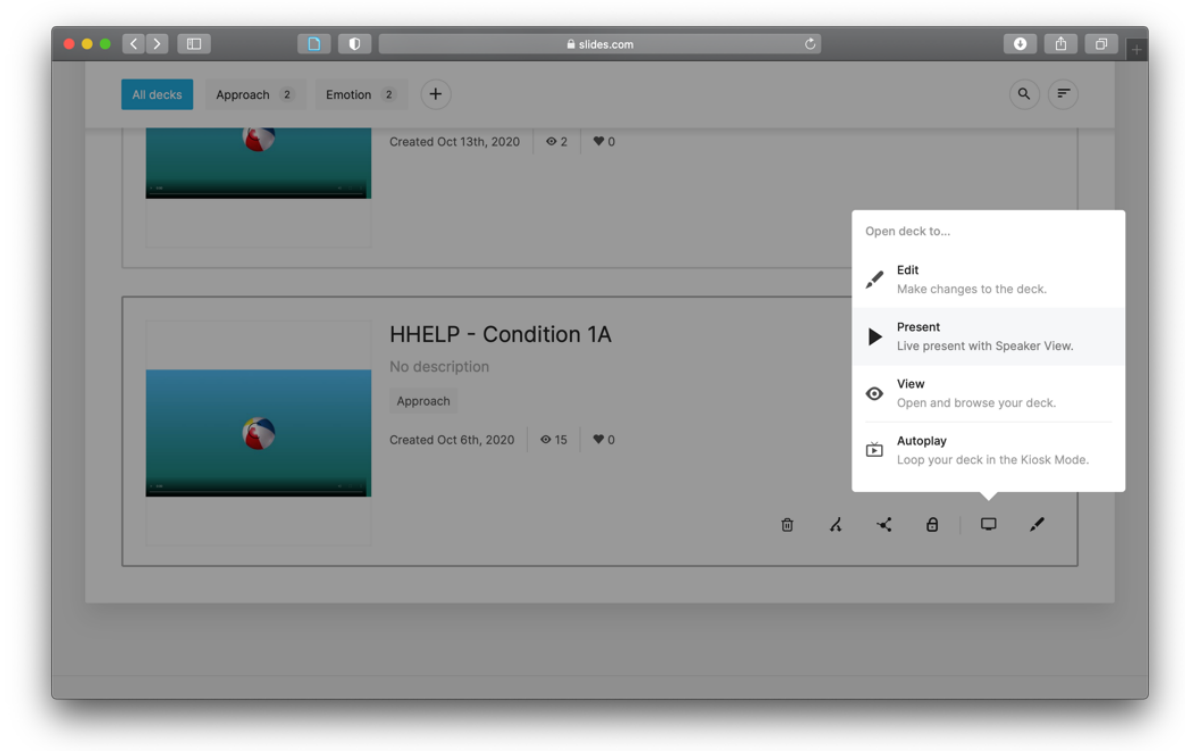

3. Right-click on the browser tab in Google Chrome. It will read Present: Name of your video. A drop-down menu will appear. Select Mute Site. As mentioned earlier, this is helpful in preventing audio interference.

4. When the participant's parent joins your Zoom call, explain how you will be presenting the stimuli. In your Slides window, copy the link provided under PRESENT LIVE, and send the parent the link in the Zoom chat. In your Slides window on your computer, click the green button that reads Start presenting.

\section{Figure 12}

Helping the parent locate the Present Live link. 


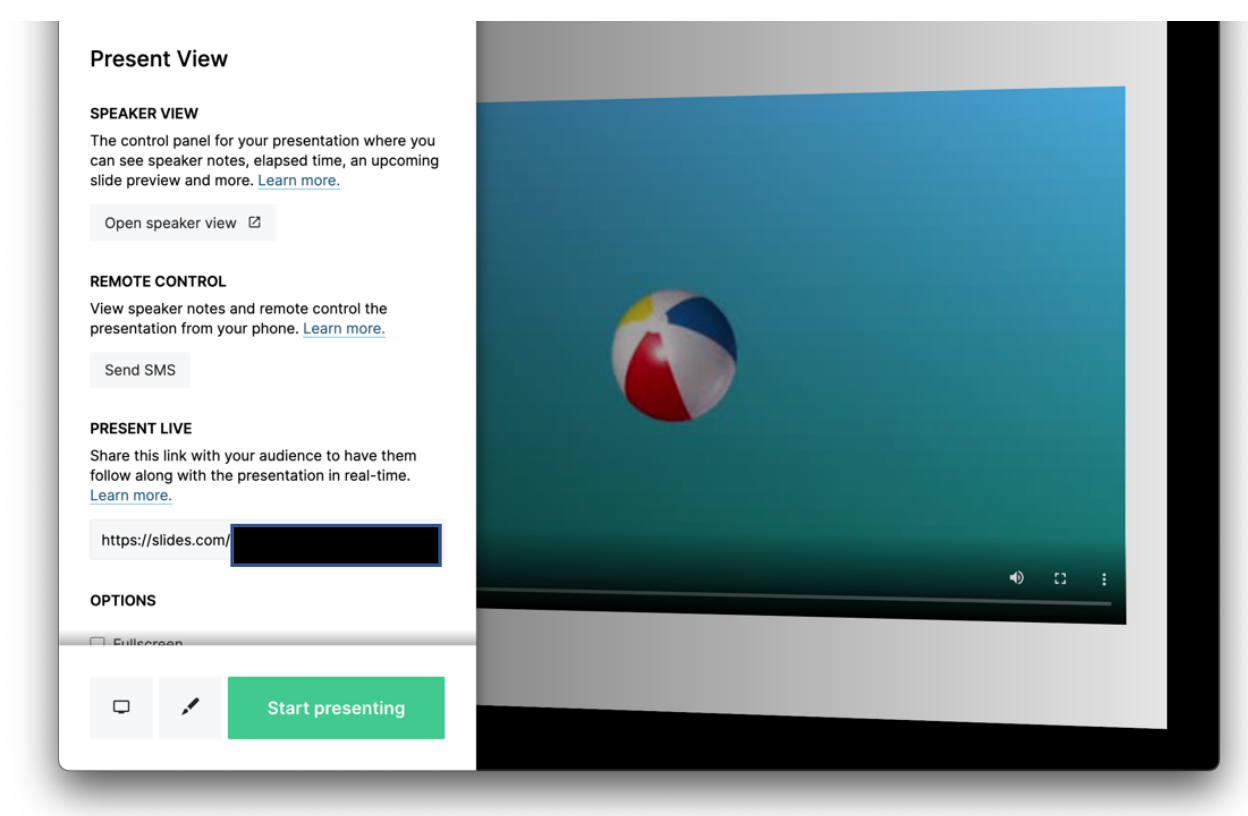

5. When the parent clicks on the link you sent them, a new browser window will open. Their screen will now have a green button that reads Continue to Presentation. Instruct them to click that green button.

6. Confirm with the parent that they now are able to see your first slide. This should match what you see on your Slides window.

7. To record video of the participant without interference from an Active Speaker, you need to Pin the participant's video. Hover over the participant's video and click the three dots that appear, then select Pin Video (Figure 13).

\section{Figure 13}

Pin the participant's video in order to record their face without interruption from another active speaker. 


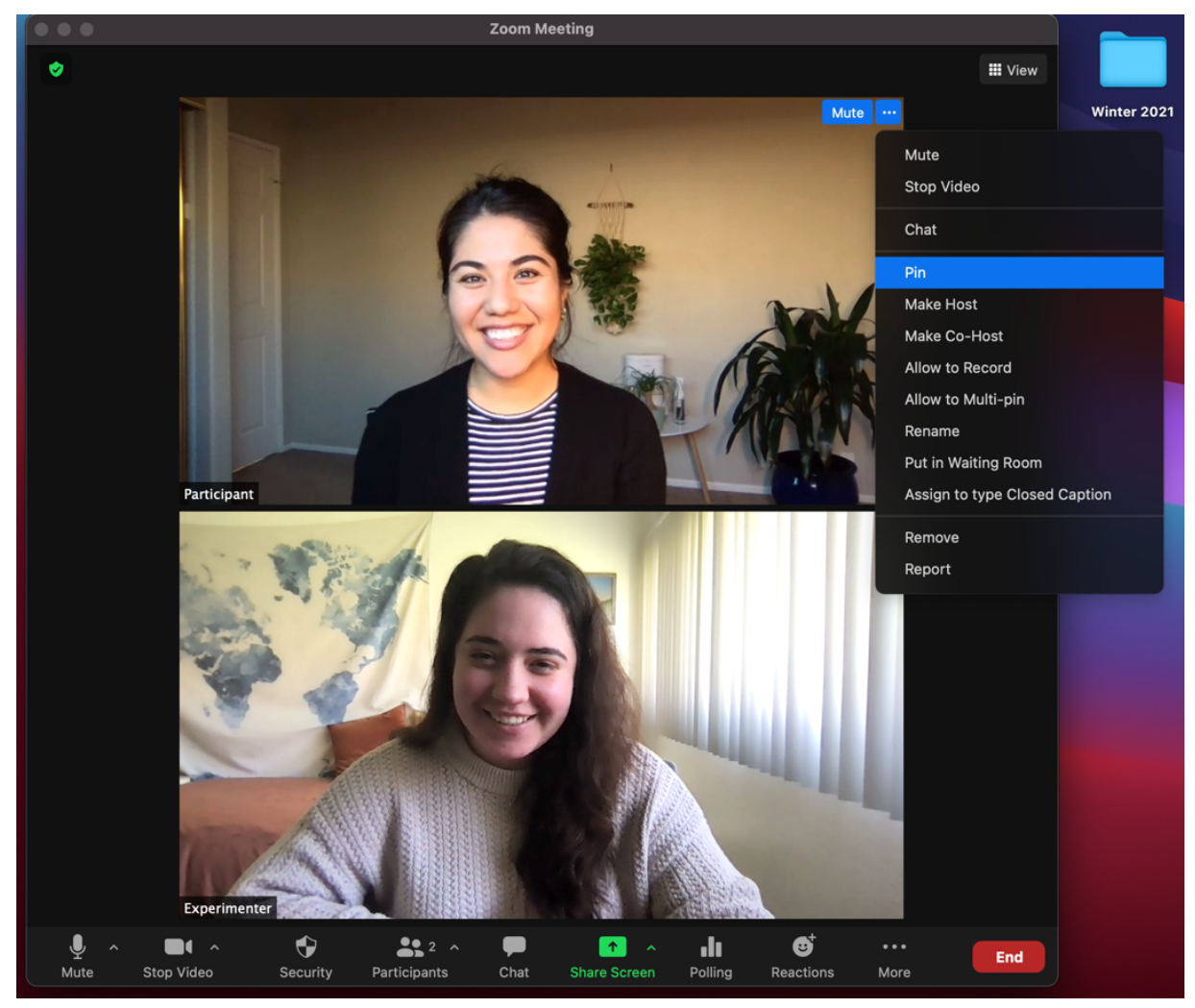

8. Allow the participant's parent to share their screen by selecting the green arrow to the right of Share Screen at the bottom of your Zoom window. A new menu will appear; click Advanced Sharing Options. In the window that appears, select AII Participants under the heading Who can share? Then exit the window (Figure 14).

9. Instruct the parent to share the window that contains the slideshow. Instruct them to select Share Screen at the bottom of their Zoom window. Ask them to select the window that contains the slides presentation, and click the box in the lower left-hand corner beside Share sound, then ask them to select Share.

\section{Figure 14}

Allow a parent to screen share by selecting the arrow beside Share Screen and selecting Advanced Sharing Options 


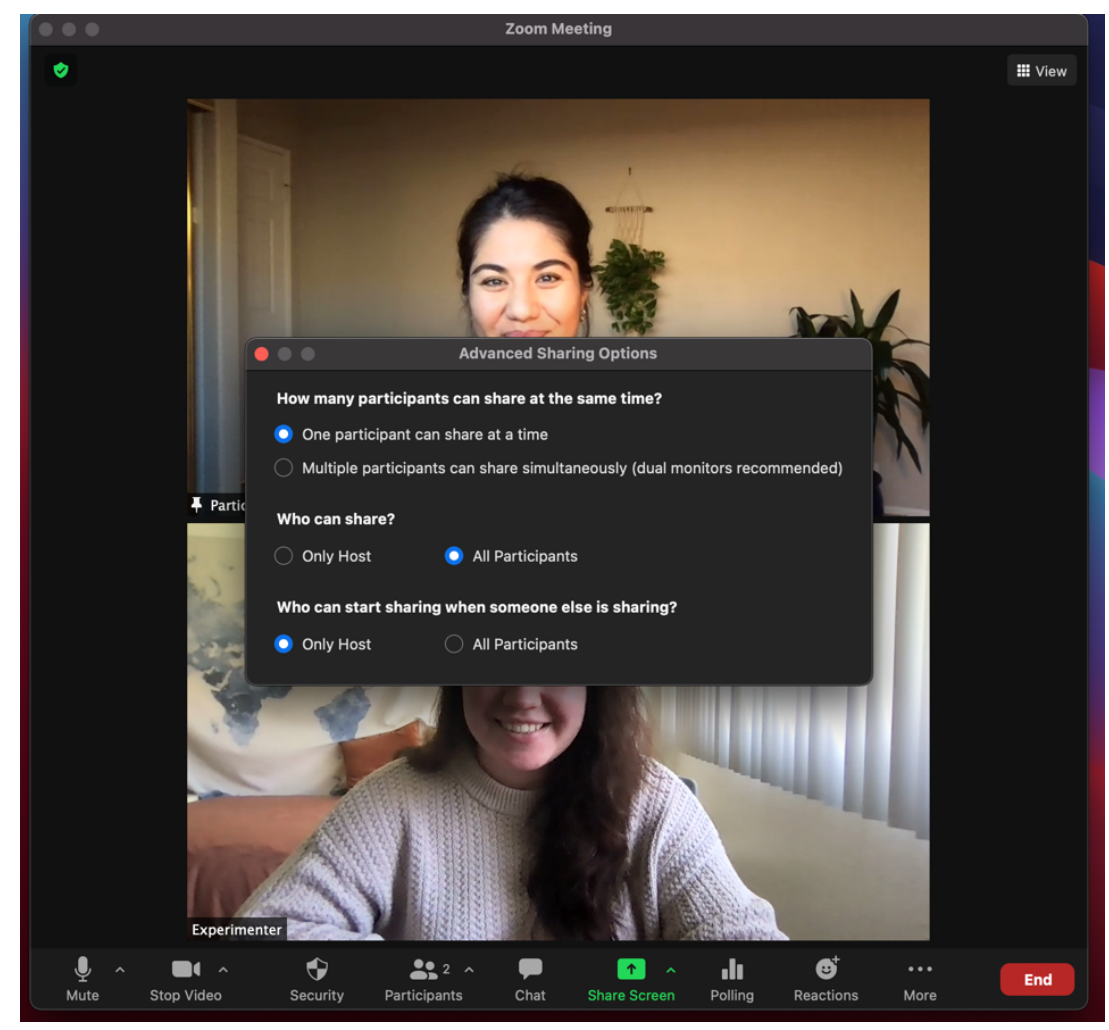

10. In your Zoom window, highlight the video of the participant by selecting View in the top right-hand corner of your screen, and selecting Side-by-Side: Speaker (Figure 15). This setting will allow you to enlarge the participant's video to make it easier to code both online and offline. 


\section{Figure 15}

Enable Side-by-Side: Speaker to view only the video of the participant

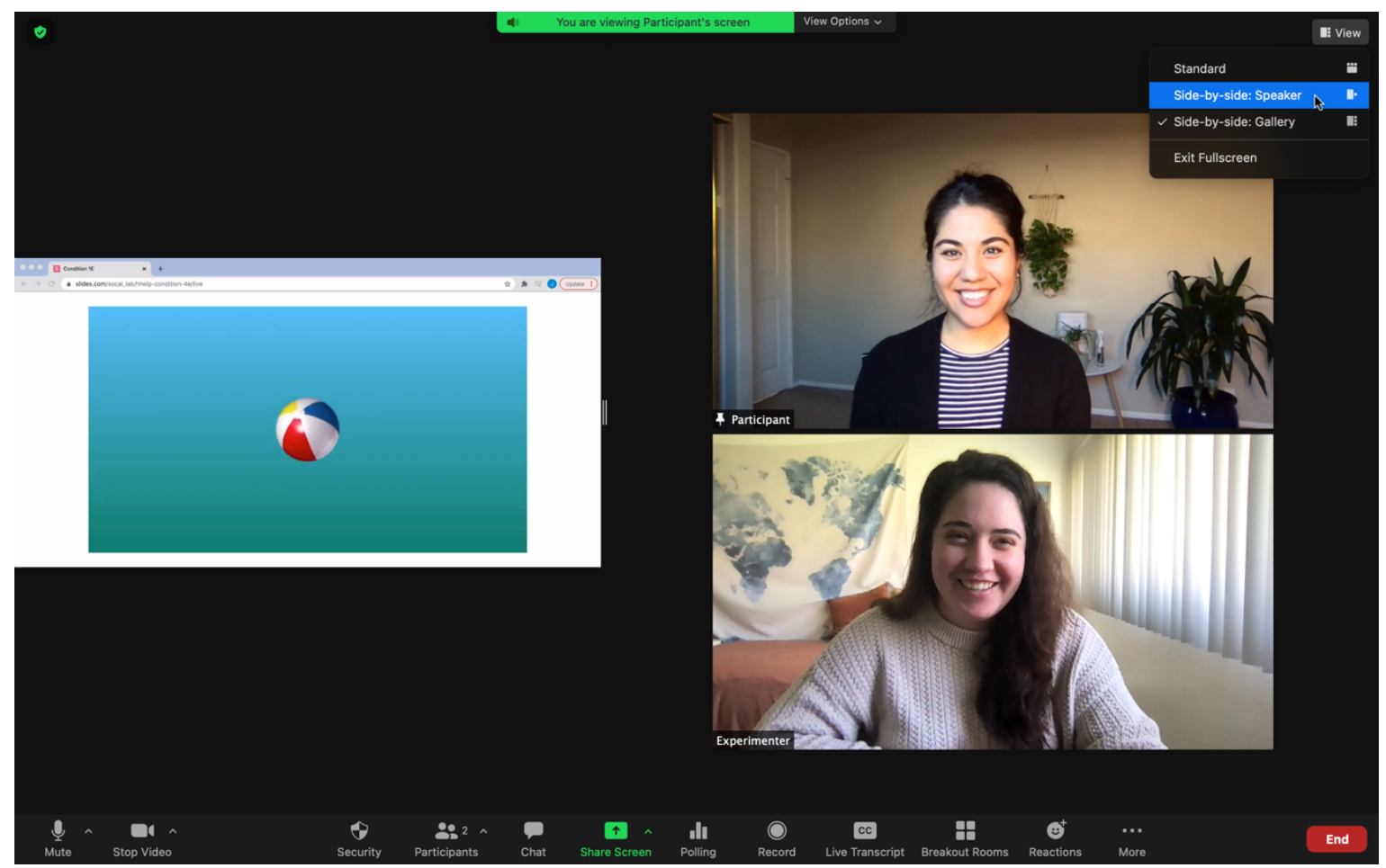

11. Instruct the parent to make the window containing the slideshow full-screen. Once they have enlarged the window, you will want them to hide their video panel and meeting controls, to ensure that nothing else is present on their screen. Ask them to select the More option on their Zoom toolbar at the top of their screen, then to select Hide Video Panel (Figure 16). Instruct them to select More again, and then to select Hide Floating Meeting Controls (Figure 17). 


\section{Figure 16}

View of participant's screen - Hide Video Panel

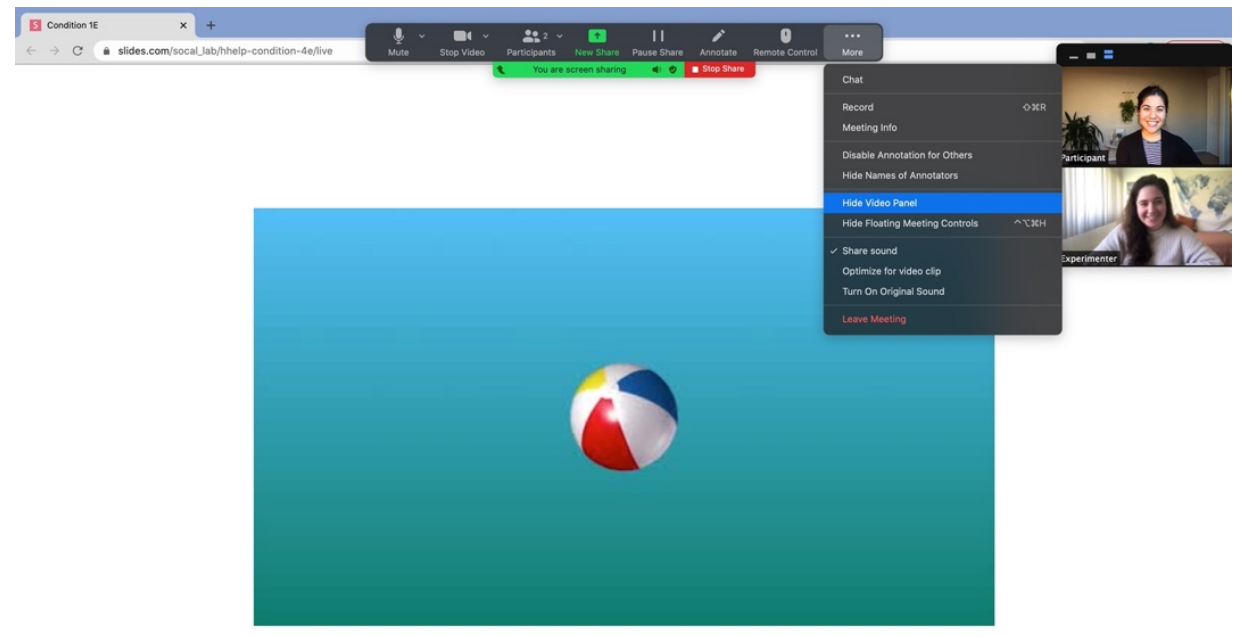

Figure 17

View of participant's screen - Hide Floating Meeting Controls

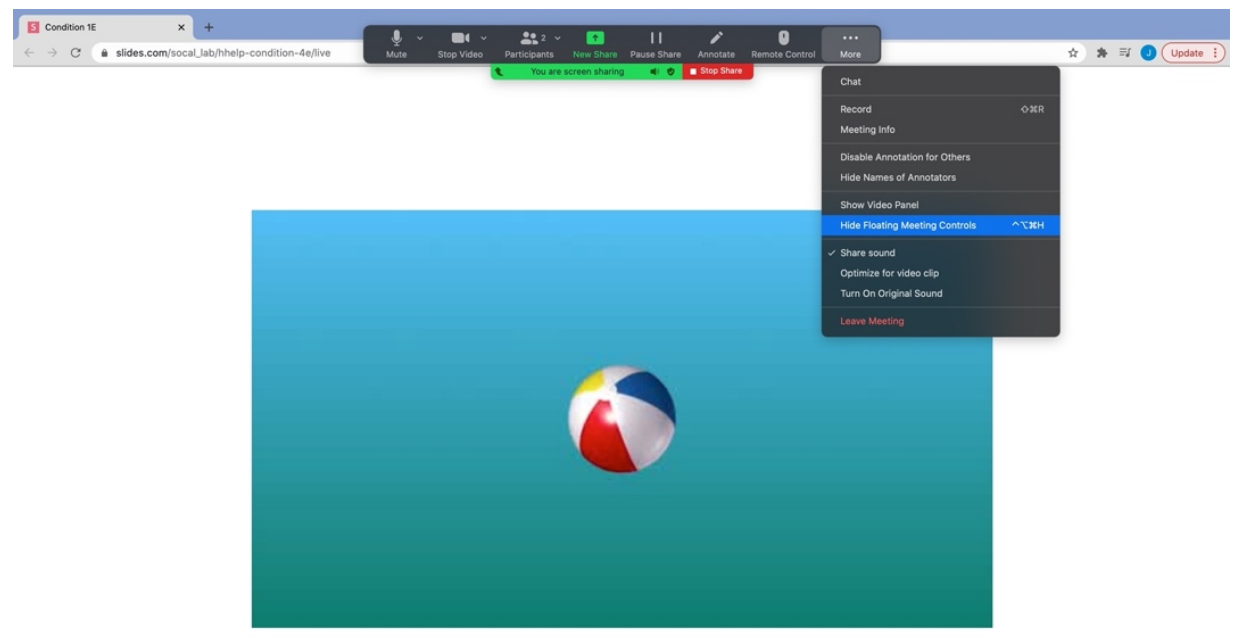

12. Zoom setup is now complete and you can start recording. Select the button that says Record at the bottom of your screen, then select Record on this Computer (Figure 18). A red light should appear on the green bar at the top of your screen. 


\section{Figure 18}

Record by clicking the circular Record button at the bottom of screen, and selecting Record on this Computer

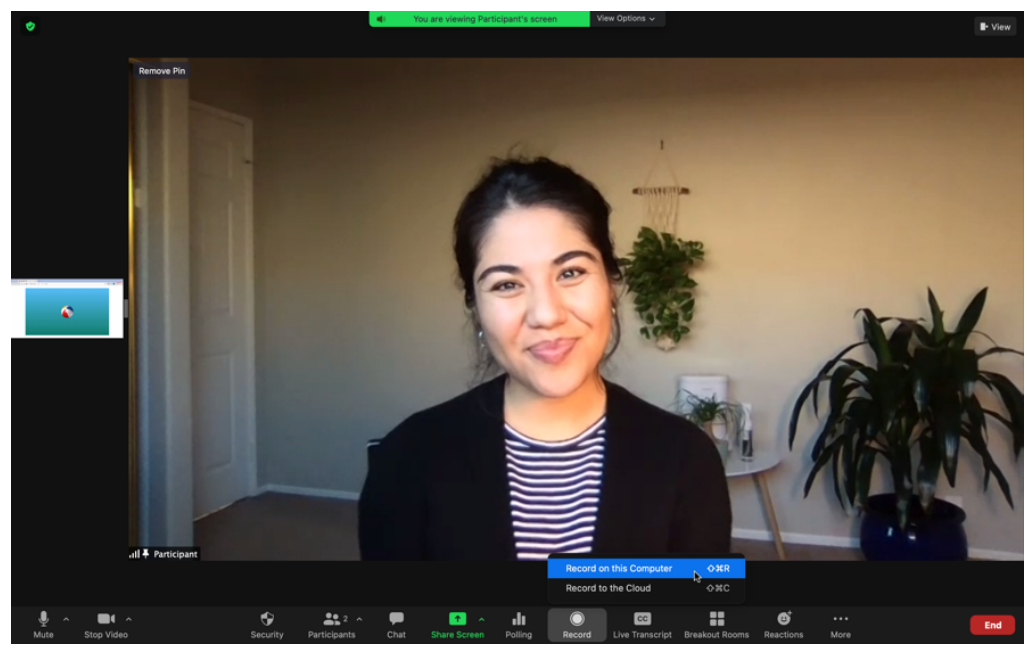

O If the video of the participant is distorted, you can stop your Zoom video (stopping your video will minimize interference and should help increase the quality of the participant's video).

○ To stop your Zoom video, select the Stop Video button in the Zoom toolbar at the top of your screen. You can see this button in Figure 18, it is the button second from the left on the toolbar at the bottom.

13. When the study ends, stop the participant's screen-sharing by selecting View Options at the top of your screen and clicking Stop Participant Screen Sharing. Stop your screen recording by clicking the Stop Recording button in the top lefthand corner. You will receive a notification that your video will be converted when the meeting ends.

- Zoom does not allow you to record twice in the same session. If you plan to test sibling participants, you must either pause the recording between participants, or save the recording, end the call, and begin a new call for the next participant.

14. When you end the call, conversion will automatically begin (Figure 19).

\section{Figure 19}

Zoom automatically converts the recording after the meeting ends. 


\section{Converting meeting recording}

You have a recording that needs to be converted before viewing $77 \%$ 